\title{
Flavan-3-ols consumption and cancer risk: a meta-analysis of epidemiologic studies
}

\author{
Lei Lei ${ }^{1,2}$, Ying Yang ${ }^{1,2}$, Hongjuan $\mathrm{He}^{1,2}$, Erfei Chen ${ }^{1,2}$, Le Du ${ }^{1,2}$, Jing Dong ${ }^{1,2}$, Jin \\ Yang ${ }^{1,2}$ \\ ${ }^{1}$ Key Laboratory of Resources Biology and Biotechnology in Western China, Ministry of Education, College of Life Science, \\ Northwest University, Xi'an 710069, China \\ ${ }^{2}$ Institute of Preventive Genomic Medicine, Xi'an 710069, China \\ Correspondence to: Jin Yang, email: yangjin@nwu.edu.cn \\ Keywords: meta-analysis, flavan-3-ols, cancer, colorectal cancer, breast cancer \\ Received: March 29, $2016 \quad$ Accepted: July 27, $2016 \quad$ Published: September 14, 2016
}

\section{ABSTRACT}

\begin{abstract}
Although numerous in vitro studies and animal model data have suggested that flavan-3-ols, the most common subclass of flavonoids in the diet, may exert protective effects against cancer, epidemiologic studies have reported inconclusive results for the association between flavan-3-ols intake and cancer risk. Therefore, we conducted this meta-analysis of epidemiologic studies to investigate the preventive effects of flavan-3-ols on various types of cancers. A total of $\mathbf{4 3}$ epidemiologic studies, consisting of 25 case-control and 18 prospective cohort studies, were included. A significant inverse association was shown between flavan-3-ols intake and the risk of overall cancer (relative risk (RR) $0.935,95 \% \mathrm{CI}: 0.891-0.981$ ). When cancer types were separately analyzed, a statistically significant protective effect of flavan-3ols consumption was observed in rectal cancer (RR 0.838, 95\%CI: 0.733-0.958), oropharyngeal and laryngeal cancer (RR $0.759,95 \% \mathrm{CI}: 0.581-0.993)$, breast (RR $0.885,95 \% \mathrm{CI}: 0.790-0.991$ ) in case-control studies and stomach cancer in women (RR 0.633, 95\%CI: 0.468-0.858). Our analysis indicates the potential benefits of flavan-3-ols in cancer prevention.
\end{abstract}

\section{INTRODUCTION}

Dietary flavonoids are a diverse range of polyphenols that are ubiquitously found in many commonly consumed vegetables, fruits, food grains, herbal remedies, beverages, and dietary supplements. Most total dietary flavonoids are flavan-3-ols (83.5\%), followed by flavanones, flavonols, anthocyanidins, flavones and isoflavones, as estimated based on the United States Department of Agriculture (USDA) database [1] and the mean daily flavonoid intake reported for adults in the United States [2]. Flavan-3-ols include (+)-catechin, $(+)$-gallocatechin, (-)-epicatechin, (-)-epigallocatechin, (-)-epicatechin 3-gallate, (-)-epigallocatechin 3-gallate, theaflavin, theaflavin 3-gallate, theaflavin 3'-gallate, theaflavin 3,3'-digallate and thearubigins [1], which are abundant in tea, wine, apples and cocoa. An extensive literature indicates that flavan-3-ols exhibit a range of biological activities both in vitro and in vivo by acting as an antioxidant, cardio-preventive, anticarcinogenic, antimicrobial, anti-viral, and neuro-protective agent [3].

To date, epidemiological studies have focused primarily on flavan-3-ols and cardiovascular disease, and meta-analyses have suggested a reduced risk of cardiovascular disease associated with flavan-3-ols intake $[4,5]$. Furthermore, when focusing on cancer preventive effects, meta-analyses of observational studies have mainly indicated an association between cancer risk and intake of other flavonoids, such as isoflavones $[6,7]$. Although numerous in vitro and animal model studies have suggested that flavan-3-ols act as anticarcinogens through antioxidant $[8,9]$ and detoxifying effects [10], modulation of the cell cycle and apoptosis [11-13], stimulation of the immune system [14] and DNA repair [15], and suppression of metastasis [16, 17] and inflammation [17-19], data from human population studies are limited and conflicting [20]. 
To our knowledge, no quantitative evaluations of the association between flavan-3-ols intake and the risk of overall cancer have been reported. Thus, we performed a quantitative meta-analysis of the currently available epidemiologic studies to estimate the effects of flavan-3ols consumption on cancer risk.

\section{RESULTS}

\section{Literature search}

We screened 658 titles or abstracts from the 3 databases, of which 178 were reviewed in full. The flow diagram is shown in Figure 1. A total of 43 studies were included in this meta-analysis, and the main reasons for excluding studies from the final review were as follows: 1 study was repeated reporting [21], 2 studies were randomized controlled trials (RCTs) [22, 23], 2 studies had an outcome of cancer mortality [24, 25], 5 studies evaluated urinary flavan-3-ols [26-30], and 2 studies evaluated plasma flavan-3-ols [31, 32].

\section{Study characteristics and quality assessment}

The main characteristics of the included studies are shown in Table 1. The types of study design were as follows: cohort studies $(n=18)$, hospital-based casecontrol studies $(\mathrm{n}=18)$, and population-based case-control studies $(\mathrm{n}=7) .13$ studies were conducted in the Americas (USA, $n=11$; Mexico, $n=1$; and Canada, $n=1$ ), 29 studies were conducted in Europe (Multiple European countries, $\mathrm{n}=5$; Italy, $\mathrm{n}=11$; Greece, $\mathrm{n}=4$; France, $\mathrm{n}=1$; UK, $\mathrm{n}=2$; Finland, $\mathrm{n}=2$; Netherlands, $\mathrm{n}=3$; and Spain, $\mathrm{n}=1$ ), and only one study was conducted in Asia (Korea, $n=1$ ). Of these studies, 7 evaluated catechins as a major flavan-3ols [33-39] and others assessed flavan-3-ols according into the USDA databases for the flavonoid content of selected foods [1] (Supplementary Table 1). These studies covered 14 different types of cancer, most of which were adjusted for a wide range of confounders, including age, total energy intake, BMI, physical activity, smoking status, alcohol intake, and total fruit and vegetable consumption. All studies used food-frequency questionnaires to measure flavan-3-ols intake.

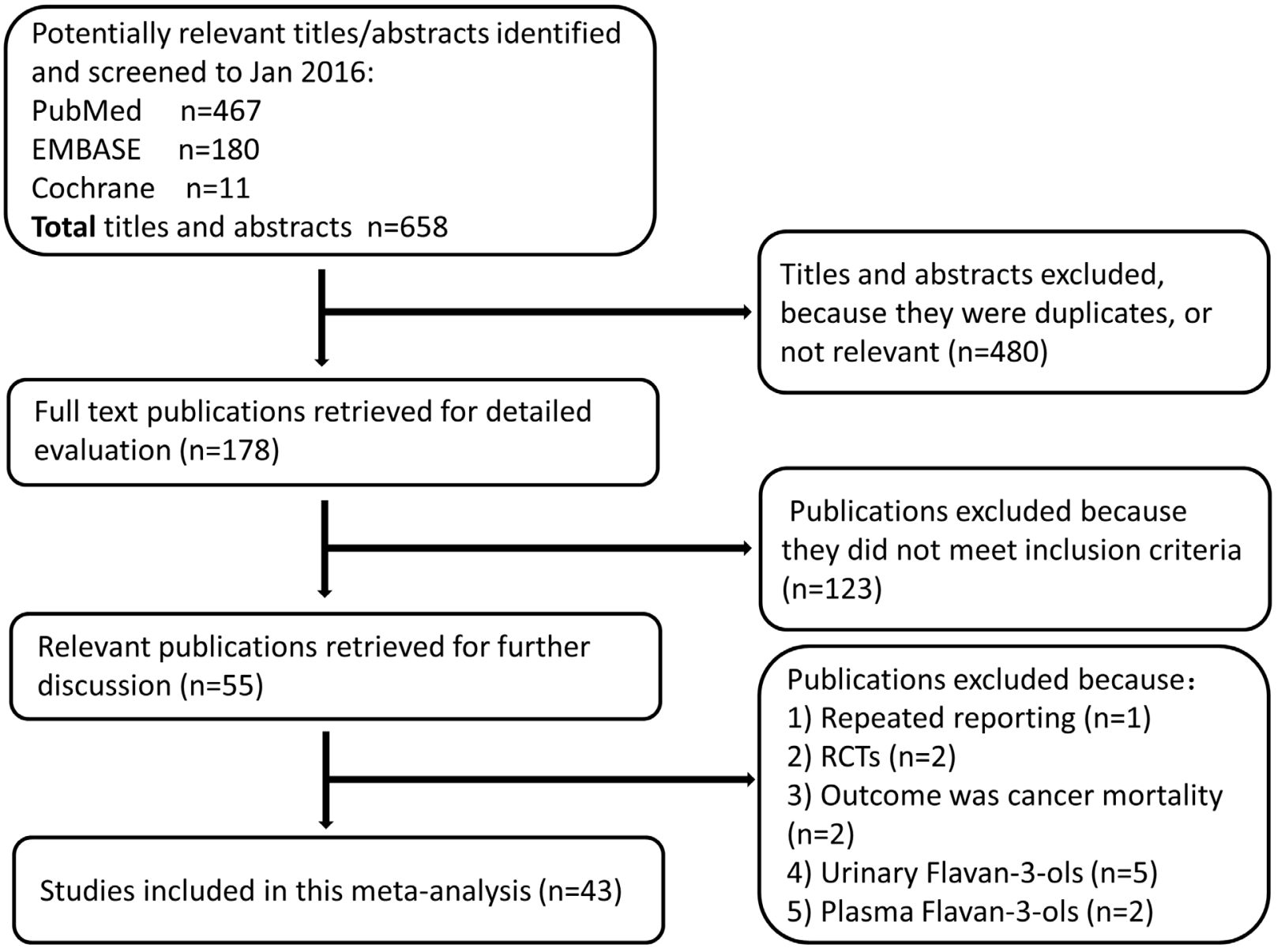

Figure 1: Flow diagram illustrating the reference search and selection in this meta-analysis (RCTs, randomized controlled trials). 
Table 1: Characteristics of included studies

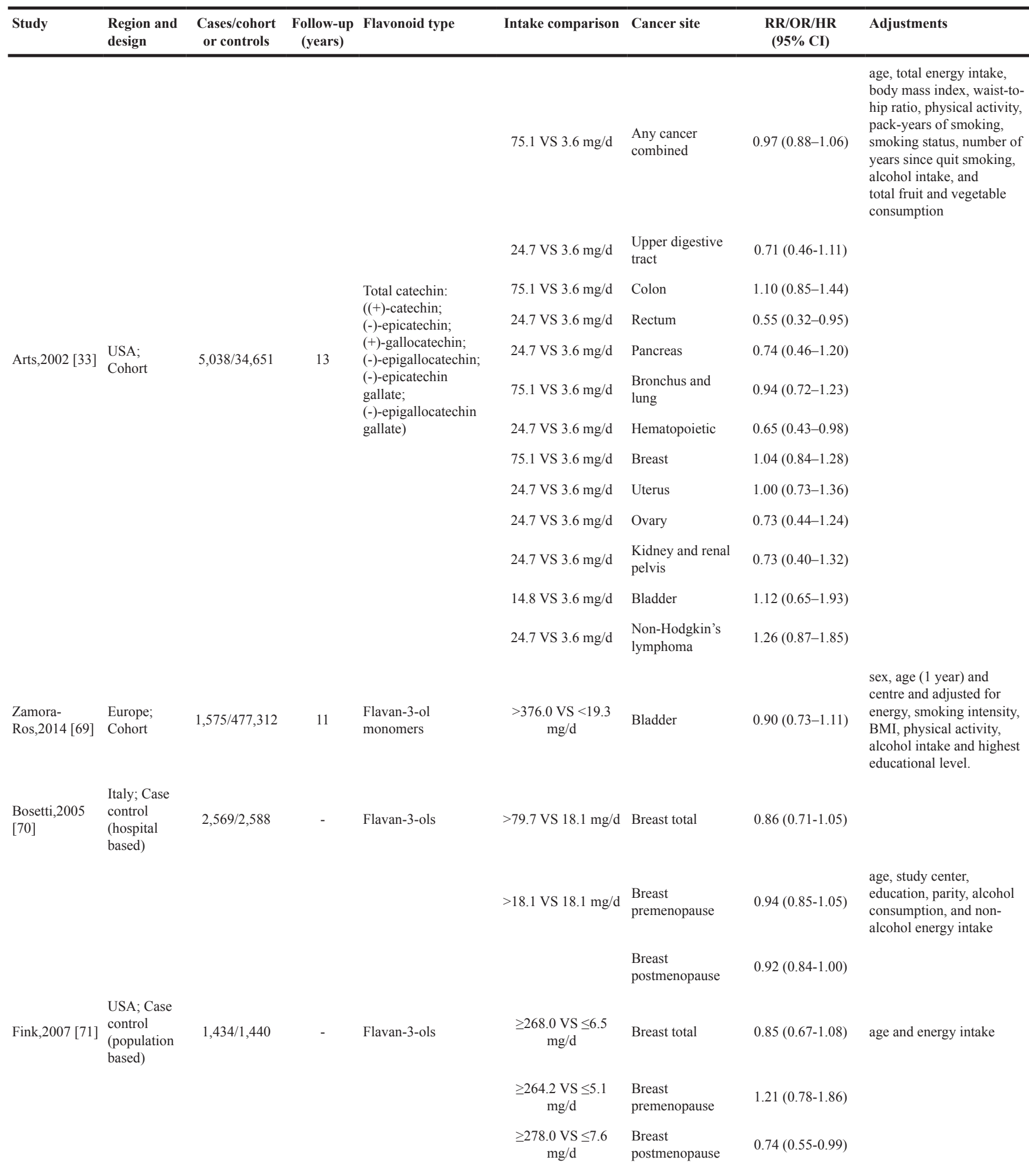

age, place of birth, parity, age at first pregnancy, age at menarche, menopausal Greece;

Peterson,2003 Case control [72] (hospital

820/1,548 - $\quad$ Flavan-3-ols Per 1 s.d. increment Breast status, body mass index, $0.93(0.78-1.11) \quad$ total energy intake, alcohol consumption, fruit and vegetable consumption and mutually between flavonoid categories

(Continued) 


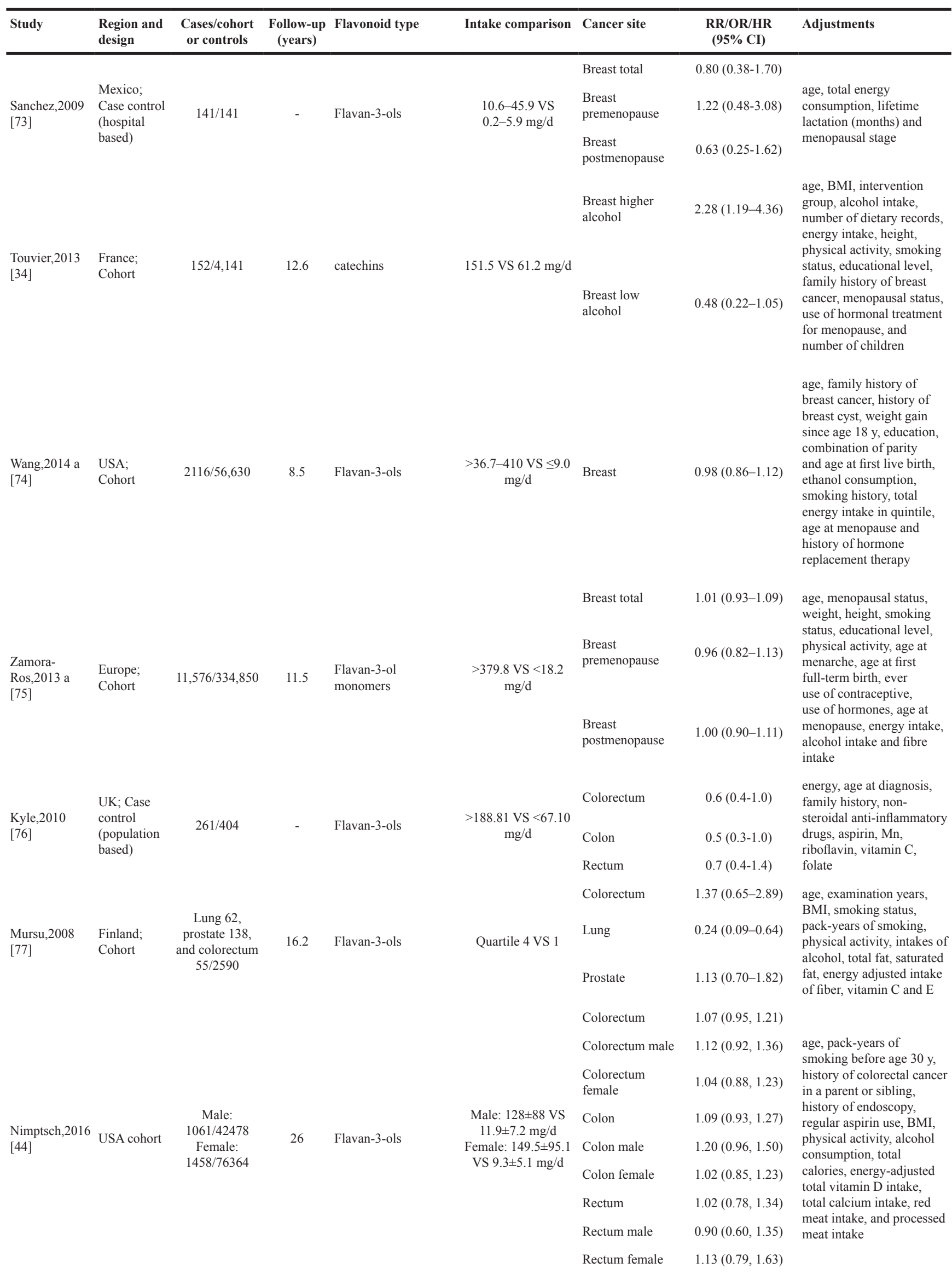

(Continued) 


\begin{tabular}{|c|c|c|c|c|c|c|c|c|}
\hline Study & $\begin{array}{l}\text { Region and } \\
\text { design }\end{array}$ & $\begin{array}{l}\text { Cases/cohort } \\
\text { or controls }\end{array}$ & $\begin{array}{l}\text { Follow-up } \\
\text { (years) }\end{array}$ & Flavonoid type & Intake comparison & Cancer site & $\begin{array}{c}\mathrm{RR} / \mathrm{OR} / \mathrm{HR} \\
(95 \% \mathrm{CI})\end{array}$ & Adjustments \\
\hline $\begin{array}{l}\text { Rossi,2006 } \\
{[78]}\end{array}$ & $\begin{array}{l}\text { Italy; Case } \\
\text { control } \\
\text { (hospital } \\
\text { based) }\end{array}$ & $1953 / 4154$ & - & Flavan-3-ols & $\begin{array}{c}>88.5 \mathrm{VS}<20.8 \\
\mathrm{mg} / \mathrm{d}\end{array}$ & Colorectum & $1.12(0.90-1.39)$ & $\begin{array}{l}\text { age, sex, study center, } \\
\text { family history, education, } \\
\text { alcohol consumption, body } \\
\text { mass index, occupational } \\
\text { physical activity, and } \\
\text { energy intake }\end{array}$ \\
\hline $\begin{array}{l}\text { Simons, } 2009 \\
{[35]}\end{array}$ & $\begin{array}{l}\text { Netherland; } \\
\text { Case-cohort }\end{array}$ & & 13.3 & $\begin{array}{l}\text { Total catechin } \\
((+) \text {-catechin; } \\
\text { (-)-epicatechin; } \\
\text { (+)-gallocatechin; } \\
\text { (-)-epigallocatechin; } \\
\text { (-)-epicatechin } \\
\text { gallate; } \\
\text { (-)-epigallocatechin } \\
\text { gallate) }\end{array}$ & $\begin{array}{l}95.9-287.3 \mathrm{VS} \\
<36.2 \mathrm{mg} / \mathrm{d}\end{array}$ & $\begin{array}{l}\text { Colon male } \\
\text { Rectum male } \\
\text { Colorectum } \\
\text { female } \\
\text { Colon female } \\
\text { Rectum female }\end{array}$ & $\begin{array}{l}0.82(0.62-1.09) \\
0.80(0.48-1.33)\end{array}$ & $\begin{array}{l}\text { age, family history of } \\
\text { colorectal cancer, smoking } \\
\text { status, alcohol intake, } \\
\text { occupational physical } \\
\text { activity at longest held job, } \\
\text { BMI and processed meat } \\
\text { intake }\end{array}$ \\
\hline $\begin{array}{l}\text { Theodoratou, } \\
2007 \text { [79] }\end{array}$ & $\begin{array}{l}\text { Scotland; } \\
\text { Case control } \\
\text { (population } \\
\text { based) }\end{array}$ & $1,456 / 1,456$ & - & Flavan-3-ols & $\begin{array}{c}>162.1 \mathrm{VS} 0-42.6 \\
\mathrm{mg} / \mathrm{d}\end{array}$ & $\begin{array}{l}\text { Colon } \\
\text { Rectum }\end{array}$ & $0.79(0.59-1.07)$ & $\begin{array}{l}\text { age, gender, family history, } \\
\text { total energy intake, total } \\
\text { fiber intake, alcohol intake, } \\
\text { NSAID intake, smoking, } \\
\text { BMI, and physical activity }\end{array}$ \\
\hline $\begin{array}{l}\text { Zamora-Ros, } \\
2013 \text { b [80] }\end{array}$ & $\begin{array}{l}\text { Spain; Case } \\
\text { control } \\
\text { (hospital } \\
\text { based) }\end{array}$ & $424 / 401$ & - & Flavan-3-ols & $\begin{array}{c}>12.9 \mathrm{VS}<4.9 \\
(\mathrm{mg} / 1,000 \text { kcal day })\end{array}$ & Colorectum & $0.78(0.45-1.34)$ & $\begin{array}{l}\text { sex, age, BMI, energy } \\
\text { intake, alcohol, fiber } \\
\text { intake, red and processed } \\
\text { meat intake, tobacco } \\
\text { consumption, physical } \\
\text { activity, regular drugs and } \\
\text { family history }\end{array}$ \\
\hline $\begin{array}{l}\text { Bobe, } 2009 \\
{[81]}\end{array}$ & $\begin{array}{l}\text { USA; Case } \\
\text { control } \\
\text { (population } \\
\text { based) }\end{array}$ & $\begin{array}{c}\text { 161EAC } \\
\text { 114ESCC/678 } \\
\text { white } \\
218 \mathrm{ESCC} / 557 \\
\text { black }\end{array}$ & - & Flavan-3-ols & \multirow[b]{2}{*}{$\begin{array}{c}\geq 130.7 \mathrm{VS} 0-10.29 \\
\mathrm{mg} / \mathrm{d}\end{array}$} & $\begin{array}{l}\text { Esophagus } \\
\text { White-ESCC }\end{array}$ & $0.95(0.36-2.52)$ & $\begin{array}{l}\text { smoking, geographical } \\
\text { area, age, BMI, hot tea, } \\
\text { hard liquor and beer, } \\
\text { moonshine consumption } \\
\text { (only for black men), } \\
\text { red wine, white wine } \\
\text { consumption (except for } \\
\text { ESCC in white men), } \\
\text { caloric intake, education } \\
\text { (only for black men) and } \\
\text { income }\end{array}$ \\
\hline $\begin{array}{l}\text { Petrick,2015 } \\
{[82]}\end{array}$ & $\begin{array}{l}\text { USA; Case } \\
\text { control } \\
\text { (population } \\
\text { based) }\end{array}$ & $\begin{array}{c}\text { EAC 274, } \\
\text { ESCC 191, } \\
\text { GCA } 248, \text { OGA } \\
341 / 622\end{array}$ & - & Flavan-3-ols & & $\begin{array}{l}\text { Esophagus EAC } \\
\text { Esophagus } \\
\text { ESCC } \\
\text { Stomach GCA } \\
\text { Stomach OGA }\end{array}$ & $\begin{array}{l}1.17(0.77-1.78) \\
1.30(0.88-1.92)\end{array}$ & $\begin{array}{l}\text { age, sex, race, geographic } \\
\text { center, cigarette smoking } \\
\text { and dietary energy intake }\end{array}$ \\
\hline $\begin{array}{l}\text { Rossi,2007 a } \\
\text { [83] }\end{array}$ & $\begin{array}{l}\text { Italy; Case } \\
\text { control } \\
\text { (hospital } \\
\text { based) }\end{array}$ & $304 / 743$ & - & Flavan-3-ols & $\begin{array}{c}>109.1 \mathrm{VS}<32.6 \\
\mathrm{mg} / \mathrm{d}\end{array}$ & $\begin{array}{l}\text { Esophagus } \\
\text { ESCC }\end{array}$ & $1.06(0.58-1.94)$ & $\begin{array}{l}\text { age, sex, study center, } \\
\text { education, alcohol } \\
\text { consumption, tobacco } \\
\text { smoking, BMI and energy } \\
\text { intake }\end{array}$ \\
\hline $\begin{array}{l}\text { Vermeulen, } \\
2013 \text { [84] }\end{array}$ & $\begin{array}{l}\text { Europe; } \\
\text { Cohort }\end{array}$ & $341 / 477,312$ & 11 & $\begin{array}{l}\text { Flavan-3-ol } \\
\text { monomers }\end{array}$ & Quartile 4 VS 1 & Esophagus & $0.86(0.58-1.27)$ & $\begin{array}{l}\text { age, sex, energy intake, } \\
\text { BMI, smoking intensity, } \\
\text { educational level, physical } \\
\text { activity, alcohol, red and } \\
\text { processed meat intake, } \\
\text { fiber, vitamin C and } \\
\text { carotenoids }\end{array}$ \\
\hline $\begin{array}{l}\text { Lagiou, } 2004 \\
\text { a [85] }\end{array}$ & $\begin{array}{l}\text { Greece; } \\
\text { Case control } \\
\text { (hospital } \\
\text { based) }\end{array}$ & $110 / 100$ & - & Flavan-3-ols & Per 1 s.d. increment & Stomach & $1.04(0.68-1.58)$ & $\begin{array}{l}\text { age, gender, place of birth, } \\
\text { BMI, height, years of } \\
\text { education, smoking habits } \\
\text { and duration of smoking, } \\
\text { alcohol consumption, total } \\
\text { energy intake, fruit and } \\
\text { vegetable consumption }\end{array}$ \\
\hline
\end{tabular}

(Continued) 


\begin{tabular}{|c|c|c|c|c|c|c|c|c|}
\hline Study & $\begin{array}{l}\text { Region and } \\
\text { design }\end{array}$ & $\begin{array}{c}\text { Cases/cohort } \\
\text { or controls }\end{array}$ & $\begin{array}{c}\text { Follow-up } \\
\text { (years) }\end{array}$ & Flavonoid type & Intake comparison & Cancer site & $\begin{array}{c}\mathrm{RR} / \mathrm{OR} / \mathrm{HR} \\
(\mathbf{9 5 \%} \mathrm{CI})\end{array}$ & Adjustments \\
\hline $\begin{array}{l}\text { Rossi,2010 } \\
{[86]}\end{array}$ & $\begin{array}{l}\text { Italy; Case } \\
\text { control } \\
\text { (hospital } \\
\text { based) }\end{array}$ & $230 / 547$ & - & Flavan-3-ols & $\begin{array}{c}>79.2 \mathrm{VS}<21.6 \\
\mathrm{mg} / \mathrm{d}\end{array}$ & Stomach & $0.75(0.45-1.23)$ & $\begin{array}{l}\text { sex, age, education, year } \\
\text { of interview, BMI, tobacco } \\
\text { smoking, and total energy } \\
\text { intake }\end{array}$ \\
\hline Woo,2014 [87] & $\begin{array}{l}\text { Korea; Case } \\
\text { control } \\
\text { (hospital } \\
\text { based) }\end{array}$ & $334 / 334$ & - & Flavan-3-ols & $39.7 \mathrm{VS} 3.2 \mathrm{mg} / \mathrm{d}$ & Stomach female & $0.78(0.41-1.49)$ & $\begin{array}{l}\text { total energy intake, } \\
\text { H. pylori, age, sex, } \\
\text { education, smoking status, } \\
\text { alcohol consumption, } \\
\text { BMI, physical activity, } \\
\text { consumption of pickled } \\
\text { vegetable and red and } \\
\text { processed meat, fruits and } \\
\text { vegetable consumption }\end{array}$ \\
\hline $\begin{array}{l}\text { Zamora-Ros, } \\
2012 \text { [88] }\end{array}$ & $\begin{array}{l}\text { Europe; } \\
\text { Cohort }\end{array}$ & $683 / 477,312$ & 11 & $\begin{array}{l}\text { Flavan-3-ol } \\
\text { monomers }\end{array}$ & $\begin{array}{c}>199.9 \mathrm{VS}<26.1 \\
\mathrm{mg} / \mathrm{d} \\
>241.6 \mathrm{VS}<21.1 \\
\mathrm{mg} / \mathrm{d}\end{array}$ & Stomach female & $0.55(0.34,0.88)$ & $\begin{array}{l}\text { center, sex, age, } \\
\text { educational level, smoking } \\
\text { status, physical activity, } \\
\text { BMI, alcohol and energy } \\
\text { intake, daily consumption } \\
\text { of fruit, vegetables, and } \\
\text { red and processed meat }\end{array}$ \\
\hline $\begin{array}{l}\text { Rossi, } 2007 \\
\text { b [89] }\end{array}$ & $\begin{array}{l}\text { Italy; Case } \\
\text { control } \\
\text { (hospital } \\
\text { based) }\end{array}$ & $805 / 2,081$ & - & Flavan-3-ols & $\begin{array}{c}>99.6 \mathrm{VS}<23.3 \\
\mathrm{mg} / \mathrm{d}\end{array}$ & Oropharynx & $0.84(0.60-1.18)$ & $\begin{array}{l}\text { sex, age, study center, } \\
\text { smoking, alcohol drinking, } \\
\text { education, BMI, and non-- } \\
\text { alcohol energy intake }\end{array}$ \\
\hline $\begin{array}{l}\text { Garavello, } \\
2007[90]\end{array}$ & $\begin{array}{l}\text { Italy; Case } \\
\text { control } \\
\text { (hospital } \\
\text { based) }\end{array}$ & $460 / 1,088$ & - & Flavan-3-ols & $\begin{array}{c}>110.4 \mathrm{VS}<31.2 \\
\mathrm{mg} / \mathrm{d}\end{array}$ & Larynx & $0.64(0.41-0.99)$ & $\begin{array}{l}\text { age, sex, study center, } \\
\text { education, alcohol } \\
\text { consumption, smoking, } \\
\text { BMI, occupational } \\
\text { physical activity and non- } \\
\text { alcohol energy intake }\end{array}$ \\
\hline $\begin{array}{l}\text { Lagiou, } 2008 \\
{[91]}\end{array}$ & $\begin{array}{l}\text { Greece; } \\
\text { Case control } \\
\text { (hospital } \\
\text { based) }\end{array}$ & $\begin{array}{c}250 \mathrm{HBV} \\
\text { and/or HCV } \\
\text { positive, } 83 \\
\mathrm{HBV} \text { and } \mathrm{HCV} \\
\text { negative/360 }\end{array}$ & - & Flavan-3-ols & $\begin{array}{c}>66.3 \mathrm{VS}<25.3 \\
\mathrm{mg} / \mathrm{d}\end{array}$ & $\begin{array}{l}\text { Liver virus } \\
\text { positive }\end{array}$ & $\begin{array}{l}1.17(0.65-2.12) \\
1.14(0.50-2.58)\end{array}$ & $\begin{array}{l}\text { gender, age, education, } \\
\text { tobacco smoking, and total } \\
\text { energy intake }\end{array}$ \\
\hline $\begin{array}{l}\text { Zamora-Ros, } \\
2013 \text { c [92] }\end{array}$ & $\begin{array}{l}\text { Europe; } \\
\text { Cohort }\end{array}$ & $191 / 477,206$ & 11 & $\begin{array}{l}\text { Flavan-3-ol } \\
\text { monomers }\end{array}$ & $\begin{array}{c}>134.8 \mathrm{VS}<28.2 \\
\mathrm{mg} / \mathrm{d}\end{array}$ & Liver & $0.65(0.39-1.07)$ & $\begin{array}{l}\text { center, sex, age, total } \\
\text { energy, education, smoke } \\
\text { intensity, alcohol lifetime } \\
\text { and alcohol baseline, BMI, } \\
\text { self-reported diabetes at } \\
\text { baseline, physical activity, } \\
\text { fiber and coffee intake }\end{array}$ \\
\hline $\begin{array}{l}\text { Arts, } 2001 \\
{[36]}\end{array}$ & $\begin{array}{l}\text { Netherland; } \\
\text { Cohort }\end{array}$ & $\begin{array}{l}96 \text { Epithelial } \\
\text { cancer, } 42 \text { lung } \\
\text { cancer/728 }\end{array}$ & 10 & $\begin{array}{l}\text { Total catechin }((+)- \\
\text { catechin; } \\
\text { (-)-epicatechin; } \\
\text { (+)-gallocatechin; } \\
\text { (-)-epigallocatechin; } \\
\text { (-)-epicatechin } \\
\text { gallate; } \\
\text { (-)-epigallocatechin } \\
\text { gallate) }\end{array}$ & $\begin{array}{c}85.8-355.4 \mathrm{VS} \\
0-49.0 \mathrm{mg} / \mathrm{d}\end{array}$ & $\begin{array}{l}\text { Epithelial cancer } \\
\text { combined }\end{array}$ & $0.92(0.41-2.07)$ & $\begin{array}{l}\text { age, physical activity, total } \\
\text { energy intake, alcohol } \\
\text { intake, smoking status, } \\
\text { pack-years of smoking, } \\
\text { BMI, coffee, fiber, vitamin } \\
\text { C, vitamin E, beta- } \\
\text { carotene }\end{array}$ \\
\hline $\begin{array}{l}\text { Christensen, } \\
2012 \text { [93] }\end{array}$ & $\begin{array}{l}\text { Canada; } \\
\text { Case control } \\
\text { (population } \\
\text { based) }\end{array}$ & $1,061 / 1,425$ & - & Flavan-3-ols & $\begin{array}{c}\text { Male: }>271.0 \mathrm{VS} \\
\quad<12.6 \mathrm{mg} / \mathrm{d} \\
\text { Female: }>249.1 \mathrm{VS} \\
\quad<8.7 \mathrm{mg} / \mathrm{d}\end{array}$ & $\begin{array}{l}\text { Lung } \\
\text { Lung smokers } \\
\text { Lung } \\
\text { nonsmokers } \\
\text { Lung male } \\
\text { Lung female }\end{array}$ & $\begin{array}{l}1.01(0.56-1.81) \\
1.18(0.84-1.67) \\
0.85(0.53-1.35)\end{array}$ & $\begin{array}{l}\text { age, sex, number of school } \\
\text { years, mean census tract } \\
\text { family income, ethnic } \\
\text { group, respondent status, } \\
\text { comprehensive smoking } \\
\text { indicator, occupational } \\
\text { exposure to carcinogens, } \\
\text { BMI, number of alcoholic } \\
\text { drinks/day and total energy } \\
\text { intake }\end{array}$ \\
\hline
\end{tabular}

(Continued) 


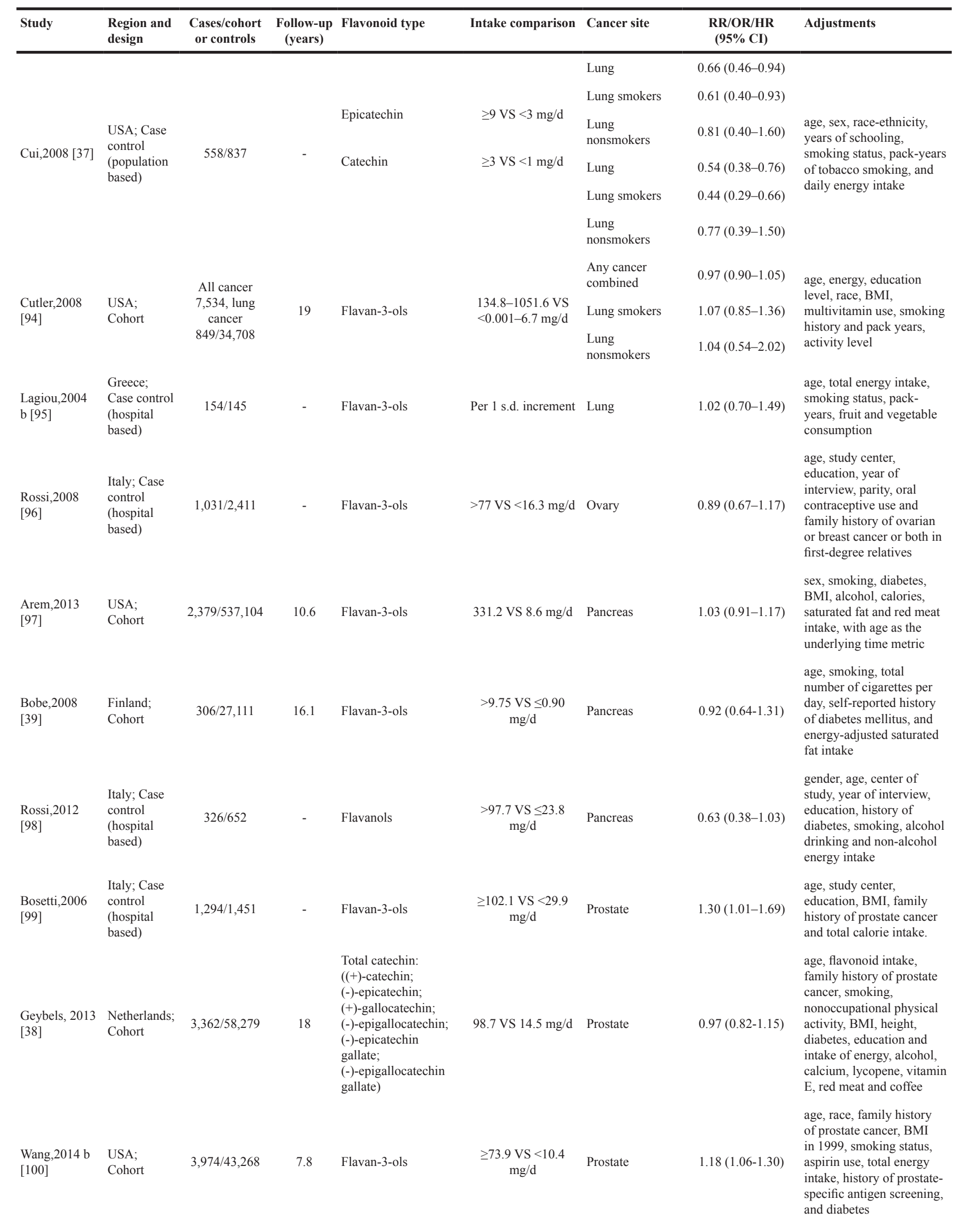

(Continued) 


\begin{tabular}{|c|c|c|c|c|c|c|c|c|}
\hline Study & $\begin{array}{l}\text { Region and } \\
\text { design }\end{array}$ & $\begin{array}{l}\text { Cases/cohort } \\
\text { or controls }\end{array}$ & $\begin{array}{l}\text { Follow-up } \\
\text { (years) }\end{array}$ & Flavonoid type & Intake comparison & Cancer site & $\begin{array}{c}\mathrm{RR} / \mathrm{OR} / \mathrm{HR} \\
(95 \% \mathrm{CI})\end{array}$ & Adjustments \\
\hline $\begin{array}{l}\text { Bosetti,2007 } \\
{[101]}\end{array}$ & $\begin{array}{l}\text { Italy; Case } \\
\text { control } \\
\text { (hospital } \\
\text { based) }\end{array}$ & $767 / 1,534$ & - & Flavan-3-ols & $\begin{array}{c}\geq 90.6 \mathrm{VS}<21.3 \\
\mathrm{mg} / \mathrm{d}\end{array}$ & Kidney & $0.77(0.56-1.06)$ & $\begin{array}{l}\text { sex, age, study center, } \\
\text { period of interview, } \\
\text { education, smoking, } \\
\text { alcohol drinking, BMI, } \\
\text { occupational physical } \\
\text { activity, family history of } \\
\text { kidney cancer, and total } \\
\text { energy intake }\end{array}$ \\
\hline $\begin{array}{l}\text { Xiao,2014 } \\
{[102]}\end{array}$ & $\begin{array}{l}\text { USA; } \\
\text { Cohort }\end{array}$ & $586 / 491,840$ & 9 & Flavan-3-ols & $\begin{array}{c}161.89-7205.21 \mathrm{VS} \\
0.00-17.16 \mathrm{mg} / \mathrm{d}\end{array}$ & Thyroid & $0.66(0.51,0.85)$ & $\begin{array}{l}\text { sex, total caloric intake, } \\
\text { smoking status, education } \\
\text { level, alcohol intake, race, } \\
\text { BMI, family history of } \\
\text { cancer }\end{array}$ \\
\hline $\begin{array}{l}\text { Rossi,2013 } \\
{[103]}\end{array}$ & $\begin{array}{l}\text { Italy; Case } \\
\text { control } \\
\text { (hospital } \\
\text { based) } \\
\text { control } \\
\text { (hospital } \\
\text { based) }\end{array}$ & $454 / 908$ & - & Flavanols & $\begin{array}{l}\text { IV vs I-III quartile } \\
\text { category }\end{array}$ & Uterus & $0.99(0.74-1.32)$ & $\begin{array}{l}\text { age, year of interview, } \\
\text { education, BMI, history of } \\
\text { diabetes, age at menarche, } \\
\text { menopausal status/age at } \\
\text { menopausal status, parity, } \\
\text { oral contraceptive use, } \\
\text { and hormone-replacement } \\
\text { therapy use }\end{array}$ \\
\hline
\end{tabular}

The quality of the included studies varied, as assessed using the Newcastle-Ottawa Scale, as shown in Tables 2 and 3. The ranges of quality scores were 8-10 in the cohort studies and 6-9 in the case-control studies. Therefore, all of the cohort studies $(n=18)$ and $84 \%$ of the case-control studies $(\mathrm{n}=21)$ were high-quality studies (studies with a score $\geq 7$ ).

\section{Overall cancer}

Our analysis of the 43 studies showed a $6.5 \%$ reduction in the risk of overall cancer with high flavan3-ols consumption (RR 0.935, 95\%CI: 0.891-0.981), as shown in Figure 2. Based on Begg's test, there was no indication of publication bias $(\mathrm{P}=0.07)$. However, the asymmetry of Egger's funnel plot suggested a possible absence of negative studies $(\mathrm{P}=0.003)$. According to the trim and fill analysis, 12 such studies were filled in to the right of the mean to make the funnel symmetrical, and the adjusted estimated effect was RR $0.987(95 \% \mathrm{CI}$ : 0.936-1.040) based on the random-effects model. In sensitivity analyses, each study was excluded one by one to determine its influence on the summary risk estimate. The RR of the remaining studies ranged from 0.928 (95\%CI: $0.882-0.977$ ) to 0.952 (95\%CI: 0.911 0.994) by omission of Zamora-Ros, 2013a and Cui, 2008 studies, respectively, without great fluctuation. Substantial heterogeneity was observed in this analysis $(\mathrm{Q}=79.002$, $\mathrm{P}=0.000, \mathrm{I}^{2}=46.837 \%$ ), which confirmed that flavan-3-ols have different effects on cancer risk, depending on the cancer site.

\section{Colorectal cancer}

As shown in Figure 3, we identified 8 studies of flavan-3-ols intake and colorectal cancer risk, which included 3 cohort studies, 1 case-cohort study, 2 population-based case-control studies and 2 hospitalbased case-control studies. The data suggested that there was no significant association between flavan-3-ols and colorectal cancer risk (RR 0.932, 95\%CI: 0.837-1.037, P for heterogeneity $\left.=0.125, \mathrm{I}^{2}=38.251 \%\right)$. No publication bias was detected with either Begg's test $(\mathrm{P}=0.536)$ or Egger's test $(\mathrm{P}=0.239)$.

When the analysis was stratified by cancer type, 7 studies suggested that flavan-3-ols were not protective in colon cancer (RR 0.983, 95\%CI: 0.863-1.120; P for heterogeneity $=0.117, \mathrm{I}^{2}=41.042 \%$ ), and 7 studies revealed a significant association between flavan-3-ols and rectal cancer (RR 0.838, 95\%CI: 0.733-0.958; P for heterogeneity $=0.562, \mathrm{I}^{2}=0 \%$ ). When the analysis was stratified by study design, an association with colorectal cancer risk was neither observed in the case-control studies (RR 0.847, 95\%CI: 0.771-1.010; 4 studies, P for heterogeneity $=0.219, \mathrm{I}^{2}=32.298 \%$ ), nor in the cohort studies (RR 0.995, 95\%CI: 0.888-1.115; 4 studies, P for heterogeneity $=0.275, \mathrm{I}^{2}=22.639 \%$ ). No publication bias was detected in these analyses.

\section{Subgroup analyses}

Table 4 shows the summary estimates of the effects of flavan-3-ols intake on cancer risk by cancer type. When the analysis was stratified by study region, the association between flavan-3-ols intake and cancer risk was observed in Europe (RR 0.926, 95\% CI: 0.880-0.974; 29 studies, $\mathrm{P}$ for heterogeneity $=0.329, \mathrm{I}^{2}=8.894 \%$ ), but not in Americas (RR 0.961, 95\%CI: 0.883-1.046; 13 studies, $\mathrm{P}$ for heterogeneity $=0.000, \mathrm{I}^{2}=72.020 \%$ ) or Asia (RR 0.73, 95\%CI: 0.45-1.18; 1 study). When the analysis was stratified by study design, protective effects of flavan-3-ols intake on breast cancer were observed in 
Table 2: Quality assessment scale score for cohort studies included in the meta-analysis ${ }^{1}$

\begin{tabular}{|c|c|c|c|c|c|c|c|c|c|c|}
\hline $\begin{array}{l}\text { First author, } \\
\text { year of } \\
\text { publication } \\
\text { (reference) }\end{array}$ & $\begin{array}{l}\text { Representa- } \\
\text { tiveness of } \\
\text { the exposed } \\
\text { cohort }\end{array}$ & $\begin{array}{l}\text { Selection } \\
\text { of the } \\
\text { unexposed } \\
\text { cohort }\end{array}$ & $\begin{array}{l}\text { Ascertain- } \\
\text { ment of } \\
\text { exposure }\end{array}$ & $\begin{array}{c}\text { Outcome } \\
\text { of interest } \\
\text { not } \\
\text { present } \\
\text { at start of } \\
\text { study }\end{array}$ & $\begin{array}{l}\text { Control } \\
\text { for } \\
\text { important } \\
\text { factor or } \\
\text { additional } \\
\text { factor }^{2}\end{array}$ & $\begin{array}{c}\text { Outcome } \\
\text { assessment }\end{array}$ & $\begin{array}{l}\text { Follow- } \\
\text { up long } \\
\text { enough } \\
\text { for } \\
\text { outcomes } \\
\text { to occur }\end{array}$ & $\begin{array}{c}\text { Adequacy of } \\
\text { follow-up of } \\
\text { cohorts }^{4}\end{array}$ & $\begin{array}{l}\text { Data analysis that } \\
\text { used an energy- } \\
\text { adjusted residual } \\
\text { or nutrient- } \\
\text { density model }\end{array}$ & $\begin{array}{c}\text { Total } \\
\text { quality } \\
\text { scores }\end{array}$ \\
\hline Arts, 2002 [33] & 幽 & 幽 & 式 & 㶦 & 论弥 & 头 & 柁 & - & is & 9 \\
\hline $\begin{array}{l}\text { Zamora- } \\
\text { Ros,2014 [69] }\end{array}$ & th & 论 & is & 论 & 论动 & is & is & - & is & 9 \\
\hline $\begin{array}{l}\text { Touvier,2013 } \\
\text { [34] }\end{array}$ & 㶦 & 为 & 为 & 头 & 动虾 & 为 & 㶦 & - & 为 & 9 \\
\hline Wang,2014 a [74] & 㶦 & 为 & 为 & 幽 & 动虾 & 头 & 㶦 & th & 为 & 10 \\
\hline $\begin{array}{l}\text { Zamora- } \\
\text { Ros,2013 a [75] }\end{array}$ & th & 式 & 为 & 论 & 论数 & 幽 & 㶦 & - & 㶦 & 9 \\
\hline Mursu,2008 [77] & 访 & 访 & - & 头 & 论弥 & 头 & is & th & 头 & 9 \\
\hline $\begin{array}{l}\text { Nimptsch,2016 } \\
{[44]}\end{array}$ & - & 式 & 论 & 论 & 动动 & 论 & 㶦 & 㶦 & - & 8 \\
\hline Simons,2009 [35] & - & th & 为 & 头 & 论弥 & 为 & 为 & is & - & 8 \\
\hline $\begin{array}{l}\text { Vermeulen,2013 } \\
{[84]}\end{array}$ & $\hat{s}$ & 头 & 㶦 & 论 & 论弥 & 头 & 访 & - & 访 & 9 \\
\hline $\begin{array}{l}\text { Zamora- } \\
\text { Ros,2012 [88] }\end{array}$ & t5 & 㶦 & 头 & 论 & 许放 & 㶦 & 头 & - & 论 & 9 \\
\hline $\begin{array}{l}\text { Zamora- } \\
\text { Ros,2013 c [92] }\end{array}$ & th & t5 & 为 & 论 & 论败 & 论 & 㶦 & - & 访 & 9 \\
\hline Arts,2001 [36] & $\hat{s}$ & 论 & 论 & 论 & 数动 & 论 & 㶦 & 论 & 约 & 10 \\
\hline Cutler,2008 [94] & 㶦 & 幽 & 头 & 头 & 动动 & - & is & 为 & 斗 & 9 \\
\hline Arem,2013 [97] & 㶦 & 头 & 论 & 论 & 论动 & 㶦 & 头 & - & 幽 & 9 \\
\hline Bobe,2008 [39] & - & 头 & 幽 & 头 & 为弥 & 幽 & 头 & - & 头 & 8 \\
\hline $\begin{array}{l}\text { Geybels,2013 } \\
\text { [38] }\end{array}$ & - & 㶦 & 论 & 论 & 论㑔 & 论 & 斗 & th & 约 & 9 \\
\hline $\begin{array}{l}\text { Wang,2014 b } \\
{[100]}\end{array}$ & t5 & 㶦 & 㶦 & 论 & 动动 & 㶦 & - & - & 论 & 8 \\
\hline Xiao,2014 [102] & 访 & th & th & th & 论弥 & 幽 & 㶦 & - & 斗 & 9 \\
\hline
\end{tabular}

1 A study could be awarded a maximum of one star for each item, while two stars for the item Control for important factor or additional factor.

2 A maximum of two stars could be awarded for this item. Studies similar to others in terms of patient characteristics (age, sex, BMI) received one star, whereas studies that controlled for other important confounders such as smoking and drinking received an additional star.

3 A cohort study with a follow-up time $>8$ y was awarded one star.

4 A cohort study with a follow-up rate $>75 \%$ was awarded one star.

the case-control studies (RR 0.885, 95\%CI: 0.790-0.991; 4 studies, $\mathrm{P}$ for heterogeneity $=0.904, \mathrm{I}^{2}=0 \%$ ) but not in the cohort studies (RR 1.009, 95\%CI: 0.946-1.075; 4 studies, $\mathrm{P}$ for heterogeneity $=0.859, \mathrm{I}^{2}=0 \%$ ). When the analysis was stratified by sex, flavan-3-ols consumption significantly reduced gastric cancer risk in women (RR 0.571, 95\%CI: 0.376-0.868; 2 studies, P for heterogeneity $\left.=0.743, \mathrm{I}^{2}=0 \%\right)$, but not in men (RR $0.928,95 \% \mathrm{CI}$ :
$0.677-1.272 ; 2$ studies, $\mathrm{P}$ for heterogeneity $=0.545, \mathrm{I}^{2}=$ $0 \%$ ). We also found a significant effect of flavan-3-ols intake on reducing the risk of rectal cancer (RR 0.838, 95\%CI: $0.733-0.958 ; 7$ studies, $\mathrm{P}$ for heterogeneity = $0.562, \mathrm{I}^{2}=0 \%$ ), oropharyngeal and laryngeal cancer (RR 0.759, 95\%CI: 0.581-0.993; 2 studies, P for heterogeneity $\left.=0.337, \mathrm{I}^{2}=0 \%\right)$, and thyroid cancer $(\mathrm{RR} 0.66,95 \% \mathrm{CI}$ : $0.51-0.85 ; 1$ study), whereas no evidence of a preventive 
Table 3: Quality assessment scale score for case-control studies included in the meta-analysis ${ }^{1}$

\begin{tabular}{|c|c|c|c|c|c|c|c|c|c|c|}
\hline $\begin{array}{l}\text { First author, year } \\
\text { of publication } \\
\text { (reference) }\end{array}$ & $\begin{array}{l}\text { Adequate } \\
\text { definition of } \\
\text { cases }\end{array}$ & $\begin{array}{l}\text { Representa- } \\
\text { tiveness of } \\
\text { cases }\end{array}$ & $\begin{array}{l}\text { Selection } \\
\text { of control } \\
\text { subjects }\end{array}$ & $\begin{array}{c}\text { Definition } \\
\text { of control } \\
\text { subjects }\end{array}$ & $\begin{array}{c}\text { Control } \\
\text { for } \\
\text { important } \\
\text { factor or } \\
\text { additional } \\
\text { factor }^{2}\end{array}$ & $\begin{array}{c}\text { Exposure } \\
\text { assessment }\end{array}$ & $\begin{array}{c}\text { Same } \\
\text { method of } \\
\text { ascertainment } \\
\text { for all } \\
\text { subjects }\end{array}$ & $\begin{array}{c}\text { Nonresponse } \\
\text { rate }^{3}\end{array}$ & $\begin{array}{c}\text { Data } \\
\text { analysis } \\
\text { that used } \\
\text { an energy- } \\
\text { adjusted } \\
\text { residual or } \\
\text { nutrient- } \\
\text { density } \\
\text { model }\end{array}$ & $\begin{array}{c}\text { Total } \\
\text { quality } \\
\text { scores }\end{array}$ \\
\hline Bosetti,2005 [70] & 㶦 & 论 & - & 柁 & 证许 & - & 㶦 & 㶦 & th & 8 \\
\hline Fink,2007 [71] & 㶦 & $\dot{w}$ & $\hat{r}$ & - & 论弥 & - & 㶦 & - & 糸 & 7 \\
\hline Peterson,2003 [72] & 㶦 & 拧 & - & - & 论弥 & - & 柁 & - & 㶦 & 6 \\
\hline Sanchez,2009 [73] & 㶦 & 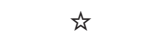 & - & is & 计放 & - & th & t5 & t5 & 8 \\
\hline Kyle,2010 [76] & 柁 & 为 & 头 & - & 论弥 & - & 访 & th & 㶦 & 8 \\
\hline Rossi,2006 [78] & 㶦 & 柁 & - & 㶦 & 论弥 & - & 㶦 & 象 & 糸 & 8 \\
\hline $\begin{array}{l}\text { Theodoratou, } 2007 \\
\text { [79] }\end{array}$ & t5 & 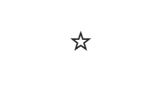 & th & - & 论动 & - & 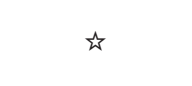 & - & t5 & 7 \\
\hline $\begin{array}{l}\text { Zamora-Ros, } \\
2013 \text { b [80] }\end{array}$ & t5 & 论 & - & 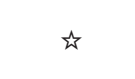 & 动动 & - & $\sqrt{5}$ & t5 & tis & 8 \\
\hline Bobe,2009 [81] & 㶦 & 㶦 & 㶦 & th & 弥访 & - & it & 㶦 & 柁 & 9 \\
\hline Petrick,2015 [82] & th & 㶦 & 㶦 & - & 论败 & - & 约 & - & 㶦 & 7 \\
\hline Rossi,2007 a [83] & 㶦 & $\sqrt{3}$ & - & 访 & 论许 & - & th & 㶦 & th & 8 \\
\hline Lagiou,2004 a [85] & 访 & 㶦 & - & - & 论弥 & - & 为 & - & 㶦 & 6 \\
\hline Rossi,2010 b [86] & 㶦 & 论 & - & 㶦 & 㶦 & - & it & 㶦 & 放 & 7 \\
\hline Woo,2014 [87] & 㶦 & 为 & - & 糸 & 论弥 & - & 访 & - & 㶦 & 7 \\
\hline Rossi,2007 b [89] & 㶦 & 论 & - & 柁 & 论许 & - & tr & 㶦 & 㶦 & 8 \\
\hline Garavello,2007 [90] & t5 & 论 & - & tit & 动动 & - & 论 & t5 & tis & 8 \\
\hline Lagiou,2008 [91] & - & 为 & - & 访 & th & - & 访 & 访 & 㶦 & 6 \\
\hline $\begin{array}{l}\text { Christensen,2012 } \\
\text { [93] }\end{array}$ & th & t5 & 㶦 & - & 论弥 & - & 㶦 & - & 㶦 & 7 \\
\hline Cui,2008 [37] & 㶦 & $\sqrt{3}$ & 论 & 柁 & 论败 & - & 㶦 & - & 㶦 & 8 \\
\hline Lagiou,2004 b [95] & 㶦 & 论 & - & - & 弥㑔 & - & 㶦 & - & th & 6 \\
\hline Rossi,2008 [96] & $\hat{s}$ & 㶦 & - & $\hat{i s}$ & 论弥 & - & 㶦 & $\hat{s}$ & - & 7 \\
\hline Rossi,2012 [98] & - & 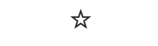 & - & 柁 & 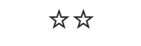 & - & t5 & 㶦 & 柁 & 7 \\
\hline Bosetti,2006 [99] & 㶦 & $\sqrt{5}$ & - & tis & 计场 & - & 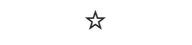 & 㶦 & th & 8 \\
\hline Bosetti,2007 [101] & 㶦 & 论 & - & th & 计场 & - & th & t5 & tit & 8 \\
\hline Rossi,2013 [103] & 弥 & 弥 & - & 论 & 论弥 & - & 头 & 柁 & - & 7 \\
\hline
\end{tabular}

1 A study could be awarded a maximum of one star for each item, while two stars for the item Control for important factor or additional factor.

2 A maximum of two stars could be awarded for this item. Studies similar to others in terms of patient characteristics (age, sex, BMI) received one star, whereas studies that controlled for other important confounders such as smoking and drinking received an additional star.

3 One star was awarded if there was no significant difference in the response rate between control and cases by chi-square test $(\mathrm{P}>0.05)$. 
Meta-analysis of flavan-3-ols and overall cancer

\begin{tabular}{|c|c|c|c|c|}
\hline \multirow{2}{*}{\multicolumn{2}{|c|}{ Study name }} & \multicolumn{3}{|c|}{ Statistics for each study } \\
\hline & & $\begin{array}{l}\text { Risk } \\
\text { ratio }\end{array}$ & $\begin{array}{c}\text { Lower } \\
\text { limit }\end{array}$ & $\begin{array}{c}\text { Upper } \\
\text { limit }\end{array}$ \\
\hline & Arem,2013 & 1.030 & 0.908 & 1.168 \\
\hline & Arts,2001 & 0.940 & 0.558 & 1.584 \\
\hline & Arts,2002 & 0.970 & 0.884 & 1.065 \\
\hline & Bobe,2008 & 0.920 & 0.643 & 1.316 \\
\hline & Bobe,2009 & 0.983 & 0.620 & 1.558 \\
\hline & Bosetti,2005 & 0.860 & 0.707 & 1.046 \\
\hline & Bosetti,2006 & 1.300 & 1.005 & 1.682 \\
\hline & Bosetti,2007 & 0.770 & 0.560 & 1.059 \\
\hline & Christensen,2012 & 1.060 & 0.809 & 1.389 \\
\hline & Cui,2008 & 0.595 & 0.464 & 0.763 \\
\hline & Cutler,2008 & 0.970 & 0.898 & 1.048 \\
\hline & Fink,2007 & 0.850 & 0.669 & 1.079 \\
\hline & Garavello,2007 & 0.640 & 0.412 & 0.995 \\
\hline & Geybels,2013 & 0.970 & 0.819 & 1.149 \\
\hline & Kyle,2010 & 0.600 & 0.368 & 0.977 \\
\hline & Lagiou,2004 a & 1.040 & 0.682 & 1.585 \\
\hline & Lagiou,2004 b & 1.020 & 0.699 & 1.488 \\
\hline & Lagiou,2008 & 1.160 & 0.718 & 1.873 \\
\hline & Mursu,2008 & 0.948 & 0.654 & 1.376 \\
\hline & Nimptsch,2016 & 1.070 & 0.948 & 1.208 \\
\hline & Peterson,2003 & 0.930 & 0.780 & 1.109 \\
\hline & Petrick,2015 & 1.123 & 0.911 & 1.383 \\
\hline & Rossi,2006 & 0.980 & 0.817 & 1.176 \\
\hline & Rossi,2007 a & 1.060 & 0.580 & 1.939 \\
\hline & Rossi,2007 b & 0.840 & 0.599 & 1.178 \\
\hline & Rossi,2008 & 0.890 & 0.673 & 1.176 \\
\hline & Rossi,2010 & 0.750 & 0.454 & 1.240 \\
\hline & Rossi,2012 & 0.630 & 0.383 & 1.037 \\
\hline & Rossi,2013 & 0.990 & 0.741 & 1.322 \\
\hline & Sanchez,2009 & 0.800 & 0.378 & 1.692 \\
\hline & Simons,2009 & 0.886 & 0.745 & 1.052 \\
\hline & Theodoratou,2007 & 0.810 & 0.650 & 1.010 \\
\hline & Touvier,2013 & 1.207 & 0.732 & 1.988 \\
\hline & Vermeulen,2013 & 0.860 & 0.581 & 1.273 \\
\hline & Wang,2014 a & 0.980 & 0.859 & 1.118 \\
\hline & Wang,2014 b & 1.180 & 1.065 & 1.307 \\
\hline & Woo,2014 & 0.730 & 0.451 & 1.182 \\
\hline & Xiao,2014 & 0.660 & 0.511 & 0.852 \\
\hline & Zamora-Ros,2012 & 0.793 & 0.595 & 1.058 \\
\hline & Zamora-Ros,2013 a & 1.010 & 0.933 & 1.093 \\
\hline & Zamora-Ros,2013 b & 0.790 & 0.489 & 1.277 \\
\hline & Zamora-Ros,2013 c & 0.650 & 0.392 & 1.077 \\
\hline & Zamora-Ros,2014 & 0.900 & 0.730 & 1.110 \\
\hline Random & & 0.935 & 0.891 & 0.981 \\
\hline
\end{tabular}
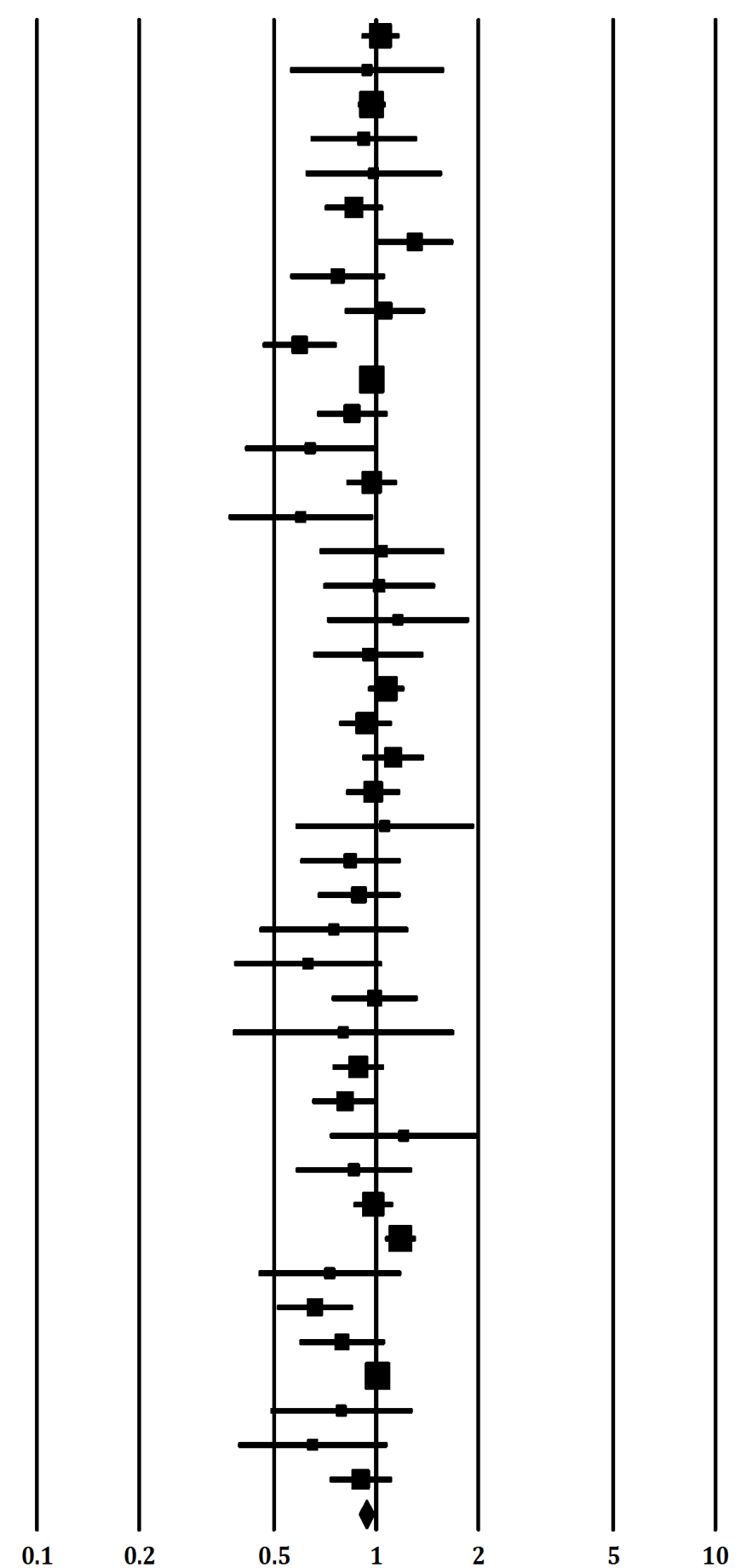

Figure 2: Association of consumption of flavan-3-ols with the risk of overall cancer. The square boxes represent study-specific estimates. The size of each box reflects the study's weight in the analysis, and the horizontal lines represent $95 \%$ CIs. The diamond indicates the combined relative risk of the analysis using the random-effects model of DerSimonian and Laird. 
Meta-analysis of flavan-3-ols and colorectal cancer

A

Model Study name

Random

$\begin{array}{lccc} & \begin{array}{c}\text { Risk } \\ \text { ratio }\end{array} & \begin{array}{c}\text { Lower } \\ \text { limit }\end{array} & \begin{array}{c}\text { Upper } \\ \text { limit }\end{array} \\ \text { Arts,2002 } & 0.964 & 0.761 & 1.222 \\ \text { Kyle,2010 } & 0.600 & 0.368 & 0.977 \\ \text { Mursu,2008 } & 1.370 & 0.650 & 2.889 \\ \text { Nimptsch,2016 } & 1.070 & 0.948 & 1.208 \\ \text { Rossi,2006 } & 0.980 & 0.817 & 1.176 \\ \text { Simons,2009 } & 0.886 & 0.745 & 1.052 \\ \text { Theodoratou,2007 } & 0.810 & 0.650 & 1.010 \\ \text { Zamora-Ros,2013b } & 0.790 & 0.489 & 1.277 \\ & 0.932 & 0.837 & 1.037\end{array}$

B

Model Study name

$\begin{array}{ccc}\begin{array}{c}\text { Risk } \\ \text { ratio }\end{array} & \begin{array}{c}\text { Lower } \\ \text { limit }\end{array} & \begin{array}{c}\text { Upper } \\ \text { limit }\end{array} \\ 1.100 & 0.845 & 1.432 \\ 0.500 & 0.258 & 0.969 \\ 1.090 & 0.933 & 1.274 \\ 1.120 & 0.901 & 1.392 \\ 0.969 & 0.796 & 1.178 \\ 0.790 & 0.587 & 1.064 \\ 0.780 & 0.452 & 1.346 \\ 0.983 & 0.863 & 1.120\end{array}$

C

Model Study name

$\begin{array}{llccc} & \begin{array}{c}\text { Risk } \\ \text { ratio }\end{array} & \begin{array}{c}\text { Lower } \\ \text { limit }\end{array} & \begin{array}{c}\text { Upper } \\ \text { limit }\end{array} \\ \text { Arts,2002 } & 0.550 & 0.319 & 0.948 \\ \text { Kyle,2010 } & 0.700 & 0.361 & 1.358 \\ \text { Nimptsch,2016 } & 1.020 & 0.778 & 1.337 \\ \text { Rossi,2006 } & 0.810 & 0.619 & 1.059 \\ \text { Simons,2009 } & 0.800 & 0.598 & 1.071 \\ \text { Theodoratou,2007 } & 0.870 & 0.615 & 1.230 \\ \text { Zamora-Ros,2013b } & 0.780 & 0.393 & 1.550 \\ \text { Random } & & 0.838 & 0.733 & 0.958\end{array}$

$\underline{\text { Risk ratio and } 95 \% \mathrm{CI}}$

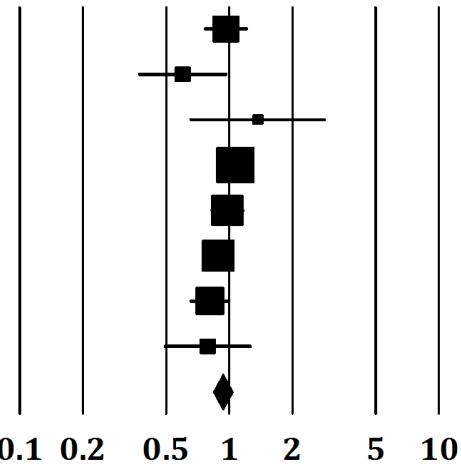

Risk ratio and $95 \% \mathrm{CI}$

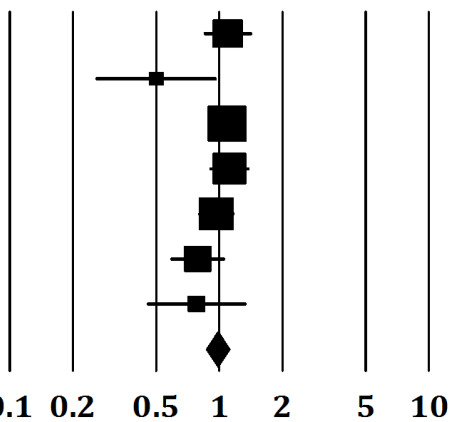

Risk ratio and $95 \%$ CI

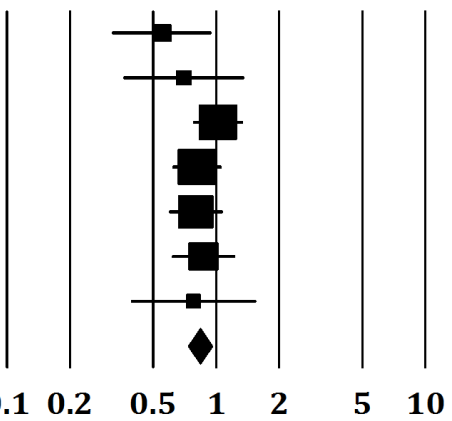

Figure 3: Effect of flavan-3-ols intake on the risk of colorectal (A), colon (B) and rectal (C) cancer. Studies were combined using the random-effects model of DerSimonian and Laird. (A) The combined relative risk of colorectal cancer was 0.932 (95\%CI: 0.8371.037, P for heterogeneity $\left.=0.125, \mathrm{I}^{2}=38.251 \%\right)$. (B) The combined relative risk of colon cancer was 0.983 (95\%CI: $0.863-1.120$; P for heterogeneity $\left.=0.117, \mathrm{I}^{2}=41.042 \%\right)$. (C) The combined relative risk of rectal cancer was 0.838 (95\%CI: $0.733-0.958$; P for heterogeneity $\left.=0.562, \mathrm{I}^{2}=0 \%\right)$. 
Table 4: Meta-analysis of flavan-3-ols consumption and cancer risk

\begin{tabular}{|c|c|c|c|c|c|c|}
\hline \multirow[t]{2}{*}{ Cancer site } & \multirow[t]{2}{*}{ No. of studies } & \multirow[t]{2}{*}{ RR $(95 \%$ CI) } & \multicolumn{3}{|c|}{ Heterogeneity test } & \multirow[t]{2}{*}{ References } \\
\hline & & & $\mathbf{Q}$ & $\mathbf{P}$ & $I^{2}(\%)$ & \\
\hline Overall cancer & 43 & $0.935(0.891-0.981)$ & 79.002 & 0.000 & 46.837 & \\
\hline Americas & 13 & $0.961(0.883-1.046)$ & 42.888 & 0.000 & 72.020 & $\begin{array}{c}{[33,37,44,71,73,74,81,} \\
82,93,94,97,100,102]\end{array}$ \\
\hline Europe & 29 & $0.926(0.880-0.974)$ & 30.733 & 0.329 & 8.894 & $\begin{array}{c}{[34-36,38,39,69,70,72,} \\
75-80,83-86,88-92,95, \\
96,98,99,101,103]\end{array}$ \\
\hline Asia & 1 & $0.73(0.45-1.18)$ & & & & {$[87]$} \\
\hline Bladder & 2 & $0.926(0.761-1.126)$ & 0.54 & 0.462 & 0 & {$[33,69]$} \\
\hline Breast & 8 & $0.977(0.924-1.033)$ & 5.213 & 0.634 & 0 & {$[33,34,70-75]$} \\
\hline premenopause & 4 & $0.957(0.878-1.043)$ & 1.494 & 0.684 & 0 & {$[70,71,73,75]$} \\
\hline postmenopause & 5 & $0.944(0.865-1.031)$ & 5.656 & 0.226 & 29.273 & {$[33,70,71,73,75]$} \\
\hline cohort & 4 & $1.009(0.946-1.075)$ & 0.76 & 0.859 & 0 & {$[33,34,74,75]$} \\
\hline Case-control & 4 & $0.885(0.790-0.991)$ & 0.566 & 0.904 & 0 & {$[70-73]$} \\
\hline Colorectum & 8 & $0.932(0.837-1.037)$ & 11.336 & 0.125 & 38.251 & {$[33,35,44,76-80]$} \\
\hline colon & 7 & $0.983(0.863-1.120)$ & 10.177 & 0.117 & 41.042 & {$[33,35,44,76,78-80]$} \\
\hline rectum & 7 & $0.838(0.733-0.958)$ & 4.857 & 0.562 & 0 & {$[33,35,44,76,78-80]$} \\
\hline cohort & 4 & $0.995(0.888-1.115)$ & 3.878 & 0.275 & 22.639 & {$[33,35,44,77]$} \\
\hline Case-control & 4 & $0.847(0.711-1.010)$ & 4.431 & 0.219 & 32.298 & {$[76,78-80]$} \\
\hline Esophagus & 4 & $0.966(0.790-1.181)$ & 1.217 & 0.976 & 0 & {$[81-84]$} \\
\hline ESCC & 3 & $0.960(0.696-1.323)$ & 0.39 & 0.942 & 0 & {$[81-83]$} \\
\hline $\mathrm{EAC}$ & 2 & $1.063(0.755-1.499)$ & 0.187 & 0.666 & 0 & {$[81,82]$} \\
\hline Stomach & 6 & $0.887(0.724-1.086)$ & 8.313 & 0.14 & 39.851 & {$[33,82,85-88]$} \\
\hline male & 2 & $0.928(0.677-1.272)$ & 0.366 & 0.545 & 0 & {$[87,88]$} \\
\hline female & 3 & $0.633(0.468-0.858)$ & 0.6 & 0.741 & 0 & {$[33,87,88]$} \\
\hline $\begin{array}{l}\text { Oropharynx and } \\
\text { Larynx }\end{array}$ & 2 & $0.759(0.581-0.993)$ & 0.92 & 0.337 & 0 & {$[89,90]$} \\
\hline Liver & 2 & $0.881(0.622-1.247)$ & 2.659 & 0.265 & 24.779 & {$[91,92]$} \\
\hline Lung & 7 & $0.854(0.667-1.094)$ & 21.753 & 0.001 & 72.417 & {$[33,36,37,77,93-95]$} \\
\hline smokers & 3 & $0.844(0.527-1.350)$ & 16.86 & 0 & 88.138 & {$[37,93,94]$} \\
\hline nonsmokers & 3 & $0.910(0.658-1.259)$ & 0.613 & 0.736 & 0 & {$[37,93,94]$} \\
\hline male & 3 & $0.702(0.299-1.648)$ & 9.05 & 0.011 & 77.9 & {$[36,77,93]$} \\
\hline female & 5 & $0.873(0.685-1.112)$ & 13.21 & 0.01 & 69.721 & {$[33,37,93-95]$} \\
\hline cohort & 4 & $0.856(0.599-1.223)$ & 8.564 & 0.036 & 64.972 & {$[33,36,77,94]$} \\
\hline Case-control & 3 & $0.854(0.572-1.275)$ & 11.128 & 0.004 & 82.027 & {$[37,93,95]$} \\
\hline Ovary & 2 & $0.851(0.666-1.088)$ & 0.436 & 0.509 & 0 & {$[33,96]$} \\
\hline Pancreas & 4 & $0.895(0.725-1.104)$ & 5.052 & 0.168 & 40.618 & {$[33,39,97,98]$} \\
\hline Prostate & 4 & $1.128(0.994-1.280)$ & 4.936 & 0.177 & 39.217 & {$[38,77,99,100]$} \\
\hline Kidney & 2 & $0.761(0.574-1.008)$ & 0.024 & 0.877 & 0 & {$[33,101]$} \\
\hline Thyroid & 1 & $0.66(0.51-0.85)$ & & & & {$[102]$} \\
\hline Uterus & 2 & $0.995(0.805-1.229)$ & 0.002 & 0.963 & 0 & {$[33,103]$} \\
\hline
\end{tabular}


effect was observed for bladder, breast, colon, esophageal, gastric, liver, lung, ovarian, pancreatic, prostate, renal, or uterine cancer.

\section{DISCUSSION}

A total of 43 epidemiologic studies on the effects of flavan-3-ols on cancer risk were identified in this review. To our knowledge, this report is the first quantitative meta-analysis to show an association between flavan-3-ols intake and the risk of overall cancer. Our data suggested that high flavan-3-ols consumption was associated with a $6.5 \%$ reduction in the risk of overall cancer. The substantial heterogeneity in the analysis confirmed that the effects of flavan-3-ols differed by cancer site. In particular, flavan-3-ols intake significantly reduced the risk of rectal cancer, oropharyngeal and laryngeal cancer, and thyroid cancer. Reductions in the risk of breast in casecontrol studies and the risk of gastric cancer in women were also observed. However, no effects on the risk of bladder, colon, esophageal, liver, lung, ovarian, pancreatic, prostate, renal, or uterine cancer were observed. In addition, the association between flavan-3-ols intake and cancer risk was observed in Europe but not in Americas or Asia. The possible explanation could be ethnic differences in food structure and genetic backgrounds.

Although this meta-analysis suggested that there was no significant association between colorectal cancer risk and flavan-3-ols consumption, several mechanisms supported by in vitro and animal studies remain biologically plausible. More specifically, in vitro studies have shown that flavan-3-ols inhibit cell proliferation and induce apoptosis in colorectal cancer cells [40-42]. In addition, animal studies have shown that catechin, a major flavan-3-ols, is able to decrease the number of intestinal tumors. One meta-analysis found an inverse association between flavan-3-ols consumption and colorectal cancer [43], which was inconsistent with our findings, likely because the analysis did not include the most recent study on the topic [44]. In particular, we found that a significant protective effect of flavan-3-ols was observed in cases of rectal, but not colon cancer. Although colon and rectal cancer are often combined, the differences between colon and rectal cancer in terms of incidence rate $[45,46]$, molecular characterization [47, 48], and risk factors [49] may explain the distinct effects of flavan-3-ols on different parts of the intestine.

No effect of flavan-3-ols on the risk of breast cancer was identified in the present study, which is similar to the findings of a meta-analysis by Hui et al [50], although that analysis included one study that evaluated urinary excretion of polyphenol metabolites (which we excluded) [28]. We excluded studies that evaluated urinary or plasma flavan-3-ols because urinary excretion of catechin metabolites has shown a weaker correlation with intake [51] and because plasma values vary substantially throughout the day [52]. When the analysis was stratified by study design, a statistically significant association between breast cancer risk and flavan-3-ols intake was observed in case-control studies only. The inconsistent conclusions between the two different study designs may be attributed to the presence of more recall and selection biases in case-control studies. For example, case subjects may have remembered their diet differently after a diagnosis of cancer compared with control subjects. Therefore, recall bias, with a misclassification of subjects, could potentially have led to a spurious association. In addition, the potential for selection bias should be considered because hospital-based control subjects who suffer from other diseases may tend to change their dietary patterns and because population-based control subjects who agree to participate in a study may be more health conscious than the general population.

Separate analyses by sex showed a significant inverse association between flavan-3-ols intake and the risk of stomach cancer in women. A Japanese pooled analysis of six cohort studies showed similar results, with green tea consumption ( $>5$ cups/day) decreasing the risk of stomach cancer in women, but not in men [53]. These gender differences could be partly explained by the regulation of sex hormones, such as estrogens. Moreover, a large prospective study reported that associations were observed between the risk of stomach cancer and age at menopause, years of fertility and years since menopause, indicating that female hormones play a protective role in stomach cancer risk [54]. Furthermore, the risk of stomach cancer was reduced in women undergoing hormone replacement therapy, and the risk increased after treatment with an antiestrogenic agent $[55,56]$. Flavan-3-ols such as epigallocatechin gallate (EGCG) and epicatechin gallate (ECG) may inhibit estradiol metabolism and enhance uptake, resulting in a moderate increase in estradiolinduced responses [57]. However, too few publications were included in this subgroup analysis to draw any definite conclusions. Further studies are needed to provide insight into the relationship between sex and flavan-3-ols intake.

Several reviews have reported statistically significant inverse associations between lung cancer risk and total dietary flavonoids intake $[58,59]$. However, no significant correlations with flavan-3-ols intake have been found $[59,60]$. Similar to the finding of our metaanalysis, no reduced risk of lung cancer was observed in prior estimates of the effects of flavan-3-ols, including in subgroup analyses by sex, smoking status, and study design. The differences in estimated effects between total dietary flavonoids and flavan-3-ols may have been caused by the contribution of other flavonoid compounds, such as quercetin, kaempferol and isoflavones [7, 60]

Similar to all meta-analyses, the present study has several limitations. First, the USDA Database for the Flavonoid Content of Selected Foods, which was 
the first to estimate flavan-3-ols consumption based on dietary data, was published in 2003 [1], so most of the investigations reviewed here were conducted after publication of this flavonoid database. Consequently, limited numbers of previous studies identifying the effects of flavan-3-ols on cancer risk could be included in the current analysis compared with meta-analyses of other flavonoids [7, 61]. Second, data from a single food-frequency questionnaire may not accurately reflect flavan-3-ols consumption, which would likely lead to nondifferential misclassification and an underestimation of the true associations. Furthermore, flavan-3-ols content lacks an accurate biomarker and is influenced by a multitude of factors, such as sunlight, season and food processing [1, 51], thus contributing to inconsistency between studies. Third, because only published studies were included in the present analysis, the analysis may have been affected by publication bias. In fact, based on Egger's test, publication bias was indicated in the analysis of overall cancer. However, the sensitivity analysis showed no change in statistically significant levels, suggesting that the observed potential bias may not have substantially affected the summary measures. Finally, substantial heterogeneity was observed in the analysis of overall cancer, which was not surprising because of the variation in the effects of flavan3 -ols on cancer risk by cancer site. Therefore, subgrouping by cancer site reduced the apparent levels of heterogeneity.

The result from random-effect linearity metaregression model suggested that there was no doseresponse relationship between flavan-3-ols and cancer risk (data not shown). Furthermore, there are no data to suggest that long term use of high dosages of flavan-3-ols is detrimental to health. However, experimental studies based on physiologic dose levels are still needed to clarify an adequate range of exposures of flavan-3-ols.

To our knowledge, this is the first meta-analysis to comprehensively assess the effects of flavan-3-ols on the risk of a range of cancers. In the future, the findings should be confirmed by more well-designed cohort or intervention studies. And the underlying mechanisms of the different preventive effects of flavan-3-ols in various cancers remain to be elucidated.

\section{MATERIALS AND METHODS}

\section{Search strategy}

The PubMed, EMBASE and Cochrane databases were searched from their inception to January 2016 using the following terms in combination: flavan-3-ols (catechin, flavonoid), cancer (neoplasm, tumor, carcinoma) and epidemiology (case-control, cohort). In addition, the references of the retrieved articles were reviewed, and the searches were not limited by language.

\section{Study selection}

An article was included in our meta-analysis if it (1) described a cohort or case-control study, (2) assessed flavan-3-ols (catechin) consumption via food intake, (3) provided a risk estimate (hazard ratio (HR), relative risk (RR), or odds ratio (OR)) and 95\% CIs for cancer incidence, and (4) provided information on adjustment for confounding factors. Studies that evaluated urinary or plasma flavan-3-ols were excluded from this analysis. When an article could not be rejected with certainty based on the title and abstract, the full text was obtained, and inclusion was assessed independently by 2 reviewers (LL and YY).

\section{Data extraction and quality assessment}

Data were extracted independently by 2 authors (LL and YY) using a common form, and differences were subsequently adjudicated. The standardized data extraction form collected the following: the study name (the first author's last name and the year of publication); the region; the study design; the type of controls for case-control studies (population-based or hospital-based controls); the sample size (cases and controls or cohort size); the follow-up time for cohort studies; the type of flavonoid intake; the comparison of flavan-3-ols intake; the type of cancer; the adjusted RR, OR or HR with 95\% CIs for the highest compared with the lowest amount of flavan-3-ols consumed; and the most completely adjustment.

The assessment of study quality used the NewcastleOttawa Scale, which is a 9-star system including three dimensions: selection; comparability; and outcome (cohort studies) or exposure (case-control studies), depending on the study type [62]. An additional point was awarded when a study analyzed an energy-adjusted residual or nutrientdensity model $[7,63]$, considering that the present metaanalysis assessed nutrient consumption and cancer risk. A study with $\geq 7$ points was considered to be a high-quality study using this 10-point system.

\section{Statistical methods}

As the incidence of cancer was relatively low over the period of time studied, the most completely adjusted OR or HR (highest versus lowest flavan-3-ols intake) was considered to be an approximation of the RR. Studies were combined using the random-effects model, in which the effect measures are $\operatorname{logRR}$ weighted using the method of DerSimonian and Laird [64]. Statistical heterogeneity among studies was estimated using $\mathrm{Q}$ and $\mathrm{I}^{2}$ statistics [65]. To assess publication bias, funnel plots were constructed, and the methods of Begg's test (rank correlation) [66] and Egger's test (linear regression) [67] were used to test funnel plot asymmetry. If a potential bias was detected $(\mathrm{P}<0.05)$, the trim and fill analysis was further used to 
examine the impact of possibly missing studies [68]. To assess whether the combined estimates could have been markedly affected by a single study, a sensitivity analysis was also conducted, in which each study was excluded one by one and the analysis was repeated based on the remaining studies. All data analyses were performed with Comprehensive Meta-Analysis Software, version v. 2.0 (CMA, Biostat, Englewood, NJ, USA).

\section{ACKNOWLEDGMENTS AND FUNDING}

We thank our laboratory members Ruitao Liu, Meng Wang, Yuan Zeng, and Xiaojuan Zhao for discussion. Lei Lei thanks Jianqing Yin for consistent support during this research. This work was supported by a grant from the National Natural Science Foundation of China (grant No. 31401081).

\section{CONFLICTS OF INTEREST}

We declared no conflicts of interest.

\section{REFERENCES}

1. Bhagwat S, Haytowitz DB, Holden JM. USDA database for the flavonoid content of selected foods, Release 3.1. Beltsville: US Department of Agriculture. 2011:03-01.

2. Chun OK, Chung SJ, Song WO. Estimated dietary flavonoid intake and major food sources of US adults. The Journal of nutrition. 2007; 137:1244-1252.

3. Aron PM, Kennedy JA. Flavan-3-ols: Nature, occurrence and biological activity. Molecular nutrition $\&$ food research. 2008; 52:79-104.

4. Hooper L, Kroon PA, Rimm EB, Cohn JS, Harvey I, Le Cornu KA, Ryder JJ, Hall WL, Cassidy A. Flavonoids, flavonoid-rich foods, and cardiovascular risk: a metaanalysis of randomized controlled trials. The American journal of clinical nutrition. 2008; 88:38-50.

5. Hooper L, Kay C, Abdelhamid A, Kroon PA, Cohn JS, Rimm EB, Cassidy A. Effects of chocolate, cocoa, and flavan-3-ols on cardiovascular health: a systematic review and meta-analysis of randomized trials. The American journal of clinical nutrition. 2012; 95:740-751.

6. Yan L, Spitznagel EL. Soy consumption and prostate cancer risk in men: a revisit of a meta-analysis. The American journal of clinical nutrition. 2009; 89:1155-1163.

7. Yang W-S, Va P, Wong M-Y, Zhang H-L, Xiang Y-B. Soy intake is associated with lower lung cancer risk: results from a meta-analysis of epidemiologic studies. The American journal of clinical nutrition. 2011; 94:1575-1583.

8. Bors W, Michel C, Stettmaier K. Electron paramagnetic resonance studies of radical species of proanthocyanidins and gallate esters. Archives of Biochemistry and Biophysics. 2000; 374:347-355.
9. Cos P, Bruyne T, Hermans N, Apers S, Berghe DV, Vlietinck A. Proanthocyanidins in health care: current and new trends. Current medicinal chemistry. 2004; 11:1345-1359.

10. Galati G, O'Brien PJ. Potential toxicity of flavonoids and other dietary phenolics: significance for their chemopreventive and anticancer properties. Free Radical Biology and Medicine. 2004; 37:287-303.

11. Chung L, Cheung T, Kong S, Fung K, Choy Y, Chan Z, Kwok T. Induction of apoptosis by green tea catechins in human prostate cancer DU145 cells. Life sciences. 2001; 68:1207-1214.

12. Horie N, Hirabayashi N, Takahashi Y, Miyauchi Y, Taguchi H, Takeishi K. Synergistic effect of green tea catechins on cell growth and apoptosis induction in gastric carcinoma cells. Biological and Pharmaceutical Bulletin. 2005; 28:574-579.

13. Tan X, Hu D, Li S, Han Y, Zhang Y, Zhou D. Differences of four catechins in cell cycle arrest and induction of apoptosis in LoVo cells. Cancer letters. 2000; 158:1-6.

14. Sharma SD, Katiyar SK. Dietary grape-seed proanthocyanidin inhibition of ultraviolet B-induced immune suppression is associated with induction of IL-12. Carcinogenesis. 2005; 27:95-102.

15. Haza AI, Morales P. Effects of $(+)$ catechin and $(-)$ epicatechin on heterocyclic amines-induced oxidative DNA damage. Journal of Applied Toxicology. 2011; 31:53-62.

16. Yance DR, Sagar SM. Targeting angiogenesis with integrative cancer therapies. Integrative cancer therapies. 2006; 5:9-29.

17. Guruvayoorappan C, Kuttan G. (+)-Catechin inhibits tumour angiogenesis and regulates the production of nitric oxide and TNF- $\alpha$ in LPS-stimulated macrophages. Innate immunity. 2008; 14:160-174.

18. Ramiro E, Franch À, Castellote C, Pérez-Cano F, Permanyer J, Izquierdo-Pulido M, Castell M. Flavonoids from Theobroma cacao down-regulate inflammatory mediators. Journal of agricultural and food chemistry. 2005; 53:8506-8511.

19. Schroeder P, Klotz L-O, Buchczyk DP, Sadik CD, Schewe T, Sies H. Epicatechin selectively prevents nitration but not oxidation reactions of peroxynitrite. Biochemical and biophysical research communications. 2001; 285:782-787.

20. Boehm K, Borrelli F, Ernst E, Habacher G, Hung SK, Milazzo S, Horneber M. Green tea (Camellia sinensis) for the prevention of cancer. The Cochrane Library. 2009.

21. Rossi M, Negri E, Parpinel M, Lagiou P, Bosetti C, Talamini R, Montella M, Giacosa A, Franceschi S, La Vecchia C. Proanthocyanidins and the risk of colorectal cancer in Italy. Cancer Causes \& Control. 2010; 21:243-250.

22. Bobe G, Sansbury LB, Albert PS, Cross AJ, Kahle L, Ashby J, Slattery ML, Caan B, Paskett E, Iber F. Dietary flavonoids and colorectal adenoma recurrence in the Polyp Prevention Trial. Cancer Epidemiology Biomarkers \& Prevention. 2008; 17:1344-1353. 
23. Bettuzzi S, Brausi M, Rizzi F, Castagnetti G, Peracchia G, Corti A. Chemoprevention of human prostate cancer by oral administration of green tea catechins in volunteers with high-grade prostate intraepithelial neoplasia: a preliminary report from a one-year proof-of-principle study. Cancer research. 2006; 66:1234-1240.

24. Fink BN, Steck SE, Wolff MS, Britton JA, Kabat GC, Gaudet MM, Abrahamson PE, Bell P, Schroeder JC, Teitelbaum SL. Dietary flavonoid intake and breast cancer survival among women on Long Island. Cancer Epidemiology Biomarkers \& Prevention. 2007; $16: 2285-2292$.

25. Kyrø C, Zamora-Ros R, Scalbert A, Tjønneland A, Dossus L, Johansen C, Bidstrup PE, Weiderpass E, Christensen J, Ward H. Pre-diagnostic polyphenol intake and breast cancer survival: the European Prospective Investigation into Cancer and Nutrition (EPIC) cohort. Breast cancer research and treatment. 2015; 154:389-401.

26. Sun C-L, Yuan J-M, Lee M-J, Yang CS, Gao Y-T, Ross RK, Mimi CY. Urinary tea polyphenols in relation to gastric and esophageal cancers: a prospective study of men in Shanghai, China. Carcinogenesis. 2002; 23:1497-1503.

27. Yuan JM, Gao YT, Yang CS, Yu MC. Urinary biomarkers of tea polyphenols and risk of colorectal cancer in the Shanghai Cohort Study. International journal of cancer. 2007; 120:1344-1350.

28. Luo J, Gao Y-T, Chow W-H, Shu X-O, Li H, Yang G, Cai Q, Rothman N, Cai H, Shrubsole MJ. Urinary polyphenols and breast cancer risk: results from the Shanghai Women's Health Study. Breast cancer research and treatment. 2010; 120:693-702.

29. Luo J, Gao YT, Chow WH, Shu Xo, Li H, Yang G, Cai Q, Li G, Rothman N, Cai H. Urinary polyphenols, glutathione S-transferases copy number variation, and breast cancer risk: Results from the Shanghai women's health study. Molecular carcinogenesis. 2012; 51:379-388.

30. Butler LM, Huang JY, Wang R, Lee M-J, Yang CS, Gao Y-T, Yuan J-M. Urinary Biomarkers of Catechins and Risk of Hepatocellular Carcinoma in the Shanghai Cohort Study. American journal of epidemiology. 2015; 181:397-405.

31. Iwasaki M, Inoue M, Sasazuki S, Miura T, Sawada N, Yamaji T, Shimazu T, Willett WC, Tsugane S. Plasma tea polyphenol levels and subsequent risk of breast cancer among Japanese women: a nested case-control study. Breast cancer research and treatment. 2010; 124:827-834.

32. Sasazuki S, Inoue M, Miura T, Iwasaki M, Tsugane S. Plasma tea polyphenols and gastric cancer risk: a casecontrol study nested in a large population-based prospective study in Japan. Cancer Epidemiology Biomarkers \& Prevention. 2008; 17:343-351.

33. Arts IC, Jacobs Jr DR, Gross M, Harnack LJ, Folsom AR. Dietary catechins and cancer incidence among postmenopausal women: the Iowa Women's Health Study (United States). Cancer Causes \& Control. 2002; $13: 373-382$
34. Touvier M, Druesne-Pecollo N, Kesse-Guyot E, Andreeva VA, Fezeu L, Galan P, Hercberg S, Latino-Martel P. Dual association between polyphenol intake and breast cancer risk according to alcohol consumption level: a prospective cohort study. Breast cancer research and treatment. 2013; 137:225-236.

35. Simons CC, Hughes LA, Arts IC, Goldbohm RA, van den Brandt PA, Weijenberg MP. Dietary flavonol, flavone and catechin intake and risk of colorectal cancer in the Netherlands Cohort Study. International journal of cancer. 2009; 125:2945-2952.

36. Arts IC, Hollman PC, Bueno de Mesquita HB, Feskens EJ, Kromhout D. Dietary catechins and epithelial cancer incidence: the Zutphen elderly study. International journal of cancer. 2001; 92:298-302.

37. Cui Y, Morgenstern H, Greenland S, Tashkin DP, Mao JT, Cai L, Cozen W, Mack TM, Lu QY, Zhang ZF. Dietary flavonoid intake and lung cancer-A population-based casecontrol study. Cancer. 2008; 112:2241-2248.

38. Geybels MS, Verhage BA, Arts IC, Van Schooten FJ, Goldbohm RA, Van Den Brandt PA. Dietary flavonoid intake, black tea consumption, and risk of overall and advanced stage prostate cancer. American journal of epidemiology. 2013; 177:1388-1398.

39. Bobe G, Weinstein SJ, Albanes D, Hirvonen T, Ashby J, Taylor PR, Virtamo J, Stolzenberg-Solomon RZ. Flavonoid intake and risk of pancreatic cancer in male smokers (Finland). Cancer Epidemiology Biomarkers \& Prevention. 2008; 17:553-562.

40. Shimizu M, Deguchi A, Lim JT, Moriwaki H, Kopelovich L, Weinstein IB. (-)-Epigallocatechin gallate and polyphenon $\mathrm{E}$ inhibit growth and activation of the epidermal growth factor receptor and human epidermal growth factor receptor-2 signaling pathways in human colon cancer cells. Clinical Cancer Research. 2005; 11:2735-2746.

41. Yang G-Y, Liao J, Kim K, Yurkow EJ, Yang CS. Inhibition of growth and induction of apoptosis in human cancer cell lines by tea polyphenols. Carcinogenesis. 1998; 19:611-616.

42. Agullo G, Gamet-Payrastre L, Fernandez Y, Anciaux N, Demigné C, Rémésy C. Comparative effects of flavonoids on the growth, viability and metabolism of a colonic adenocarcinoma cell line (HT29 cells). Cancer letters. 1996; 105:61-70.

43. Woo HD, Kim J. Dietary flavonoid intake and risk of stomach and colorectal cancer. World journal of gastroenterology. 2013; 19:1011.

44. Nimptsch K, Zhang X, Cassidy A, Song M, O’Reilly ÉJ, Lin JH, Pischon T, Rimm EB, Willett WC, Fuchs CS. Habitual intake of flavonoid subclasses and risk of colorectal cancer in 2 large prospective cohorts. The American journal of clinical nutrition. 2016; 103:184-191.

45. Greenlee RT, Murray T, Bolden S, Wingo PA. Cancer statistics, 2000. CA Cancer J Clin. 2000; 50:7-33. 
46. Jemal A, Siegel R, Ward E, Hao Y, Xu J, Murray T, Thun MJ. Cancer statistics, 2008. CA Cancer J Clin. 2008; 58:71-96.

47. Frattini M, Balestra D, Suardi S, Oggionni M, Alberici P, Radice P, Costa A, Daidone MG, Leo E, Pilotti S. Different genetic features associated with colon and rectal carcinogenesis. Clinical Cancer Research. 2004; 10:4015-4021.

48. Kapiteijn E, Liefers G, Los L, Klein Kranenbarg E, Hermans J, Tollenaar R, Moriya Y, van de Velde C, van Krieken J. Mechanisms of oncogenesis in colon versus rectal cancer. The Journal of pathology. 2001; 195:171-178.

49. Wei EK, Giovannucci E, Wu K, Rosner B, Fuchs CS, Willett WC, Colditz GA. Comparison of risk factors for colon and rectal cancer. International journal of cancer. 2004; 108:433-442.

50. Hui C, Qi X, Qianyong Z, Xiaoli P, Jundong Z, Mantian M. Flavonoids, flavonoid subclasses and breast cancer risk: a meta-analysis of epidemiologic studies. PLoS One. 2013; 8:e54318.

51. Pérez-Jiménez J, Hubert J, Hooper L, Cassidy A, Manach C, Williamson G, Scalbert A. Urinary metabolites as biomarkers of polyphenol intake in humans: a systematic review. The American journal of clinical nutrition. 2010:ajcn. 29924.

52. Manach C, Williamson G, Morand C, Scalbert A, Rémésy C. Bioavailability and bioefficacy of polyphenols in humans. I. Review of 97 bioavailability studies. The American journal of clinical nutrition. 2005; 81:230S-242S.

53. Inoue M, Sasazuki S, Wakai K, Suzuki T, Matsuo K, Shimazu T, Tsuji I, Tanaka K, Mizoue T, Nagata C. Green tea consumption and gastric cancer in Japanese: a pooled analysis of six cohort studies. Gut. 2009.

54. Freedman ND, Chow W-H, Gao Y-T, Shu X-O, Ji B-T, Yang G, Lubin JH, Li H-L, Rothman N, Zheng W. Menstrual and reproductive factors and gastric cancer risk in a large prospective study of women. Gut. 2007; 56:1671-1677.

55. Lindblad M, Rodriguez LG, Chandanos E, Lagergren J. Hormone replacement therapy and risks of oesophageal and gastric adenocarcinomas. British journal of cancer. 2006; 94:136-141.

56. Camargo MC, Goto Y, Zabaleta J, Morgan DR, Correa P, Rabkin CS. Sex hormones, hormonal interventions, and gastric cancer risk: a meta-analysis. Cancer Epidemiology Biomarkers \& Prevention. 2011.

57. Goodin M, Fertuck K, Zacharewski T, Rosengren R. Estrogen receptor-mediated actions of polyphenolic catechins in vivo and in vitro. Toxicological Sciences. 2002; 69:354-361.

58. Romagnolo DF, Selmin OI. Flavonoids and cancer prevention: a review of the evidence. Journal of nutrition in gerontology and geriatrics. 2012; 31:206-238.
59. Tang N-P, Zhou B, Wang B, Yu R-B, Ma J. Flavonoids intake and risk of lung cancer: a meta-analysis. Japanese journal of clinical oncology. 2009; 39:352-359.

60. Woo HD, Kim J. Dietary flavonoid intake and smokingrelated cancer risk: a meta-analysis. PLoS One. 2013; 8:e75604.

61. Trock BJ, Hilakivi-Clarke L, Clarke R. Meta-analysis of soy intake and breast cancer risk. Journal of the National Cancer Institute. 2006; 98:459-471.

62. Wells G, Shea B, O'connell D, Peterson J, Welch V, Losos M, Tugwell P. (2000). The Newcastle-Ottawa Scale (NOS) for assessing the quality of nonrandomised studies in meta-analyses.

63. Willett WC, Howe GR, Kushi LH. Adjustment for total energy intake in epidemiologic studies. The American journal of clinical nutrition. 1997; 65:1220S-1228S.

64. DerSimonian R, Laird N. Meta-analysis in clinical trials. Controlled clinical trials. 1986; 7:177-188.

65. Higgins J, Thompson SG. Quantifying heterogeneity in a meta-analysis. Statistics in medicine. 2002; 21:1539-1558.

66. Begg CB, Mazumdar M. Operating characteristics of a rank correlation test for publication bias. Biometrics. 1994:1088-1101.

67. Egger M, Smith GD, Schneider M, Minder C. Bias in metaanalysis detected by a simple, graphical test. Bmj. 1997; 315:629-634.

68. Duval S, Tweedie R. Trim and fill: a simple funnel-plotbased method of testing and adjusting for publication bias in meta-analysis. Biometrics. 2000; 56:455-463.

69. Zamora-Ros R, Sacerdote C, Ricceri F, Weiderpass E, Roswall N, Buckland G, St-Jules D, Overvad K, Kyrø $\mathrm{C}$, Fagherazzi G. Flavonoid and lignan intake in relation to bladder cancer risk in the European Prospective Investigation into Cancer and Nutrition (EPIC) study. British journal of cancer. 2014.

70. Bosetti C, Spertini L, Parpinel M, Gnagnarella P, Lagiou P, Negri E, Franceschi S, Montella M, Peterson J, Dwyer J. Flavonoids and breast cancer risk in Italy. Cancer Epidemiology Biomarkers \& Prevention. 2005; 14:805-808.

71. Fink BN, Steck SE, Wolff MS, Britton JA, Kabat GC, Schroeder JC, Teitelbaum SL, Neugut AI, Gammon MD. Dietary flavonoid intake and breast cancer risk among women on Long Island. American journal of epidemiology. 2007; 165:514-523.

72. Peterson J, Lagiou P, Samoli E, Lagiou A, Katsouyanni K, La Vecchia C, Dwyer J, Trichopoulos D. Flavonoid intake and breast cancer risk: a case-control study in Greece. British journal of cancer. 2003; 89:1255-1259.

73. Torres-Sanchez L, Galvan-Portillo M, Wolff MS, LopezCarrillo L. Dietary consumption of phytochemicals and breast cancer risk in Mexican women. Public health nutrition. 2009; 12:825-831. 
74. Wang Y, Gapstur SM, Gaudet MM, Peterson JJ, Dwyer JT, McCullough ML. Evidence for an Association of Dietary Flavonoid Intake with Breast Cancer Risk by Estrogen Receptor Status Is Limited. The Journal of nutrition. 2014; 144:1603-1611.

75. Zamora-Ros R, Ferrari P, González CA, Tjønneland A, Olsen A, Bredsdorff L, Overvad K, Touillaud M, Perquier F, Fagherazzi G. Dietary flavonoid and lignan intake and breast cancer risk according to menopause and hormone receptor status in the European Prospective Investigation into Cancer and Nutrition (EPIC) Study. Breast cancer research and treatment. 2013; 139:163-176.

76. Kyle JA, Sharp L, Little J, Duthie GG, McNeill G. Dietary flavonoid intake and colorectal cancer: a case-control study. British journal of nutrition. 2010; 103:429-436.

77. Mursu J, Nurmi T, Tuomainen TP, Salonen JT, Pukkala E, Voutilainen S. Intake of flavonoids and risk of cancer in Finnish men: the Kuopio Ischaemic Heart Disease Risk Factor Study. International journal of cancer. 2008; 123:660-663.

78. Rossi M, Negri E, Talamini R, Bosetti C, Parpinel M, Gnagnarella P, Franceschi S, Dal Maso L, Montella M, Giacosa A. Flavonoids and colorectal cancer in Italy. Cancer Epidemiology Biomarkers \& Prevention. 2006; 15:1555-1558.

79. Theodoratou E, Kyle J, Cetnarskyj R, Farrington SM, Tenesa A, Barnetson R, Porteous M, Dunlop M, Campbell H. Dietary flavonoids and the risk of colorectal cancer. Cancer Epidemiology Biomarkers \& Prevention. 2007; 16:684-693.

80. Zamora-Ros R, Not C, Guinó E, Luján-Barroso L, García RM, Biondo S, Salazar R, Moreno V. Association between habitual dietary flavonoid and lignan intake and colorectal cancer in a Spanish case-control study (the Bellvitge Colorectal Cancer Study). Cancer Causes \& Control. 2013; 24:549-557.

81. Bobe G, Peterson JJ, Gridley G, Hyer M, Dwyer JT, Brown LM. Flavonoid consumption and esophageal cancer among black and white men in the United States. International journal of cancer. 2009; 125:1147-1154.

82. Petrick J, Steck S, Bradshaw P, Trivers K, Abrahamson P, Engel L, He K, Chow W, Mayne S, Risch H. Dietary intake of flavonoids and oesophageal and gastric cancer: incidence and survival in the United States of America (USA). British journal of cancer. 2015.

83. Rossi M, Garavello W, Talamini R, La Vecchia C, Franceschi S, Lagiou P, Zambon P, Maso LD, Bosetti C, Negri E. Flavonoids and risk of squamous cell esophageal cancer. International journal of cancer. 2007; 120:1560-1564.

84. Vermeulen E, Zamora-Ros R, Duell EJ, Luján-Barroso L, Boeing H, Aleksandrova K, Bueno-de-Mesquita HB, Scalbert A, Romieu I, Fedirko V. Dietary flavonoid intake and esophageal cancer risk in the European prospective investigation into cancer and nutrition cohort. American journal of epidemiology. 2013; 178:570-581.

85. Lagiou P, Samoli E, Lagiou A, Peterson J, Tzonou A, Dwyer J, Trichopoulos D. Flavonoids, vitamin C and adenocarcinoma of the stomach. Cancer Causes \& Control. 2004; 15:67-72.

86. Rossi M, Rosato V, Bosetti C, Lagiou P, Parpinel M, Bertuccio P, Negri E, La Vecchia C. Flavonoids, proanthocyanidins, and the risk of stomach cancer. Cancer Causes \& Control. 2010; 21:1597-1604.

87. Woo HD, Lee J, Choi IJ, Kim CG, Lee JY, Kwon O, Kim J. Dietary Flavonoids and Gastric Cancer Risk in a Korean Population. Nutrients. 2014; 6:4961-4973.

88. Zamora-Ros R, Agudo A, Luján-Barroso L, Romieu I, Ferrari P, Knaze V, Bueno-de-Mesquita HB, Leenders M, Travis RC, Navarro C. Dietary flavonoid and lignan intake and gastric adenocarcinoma risk in the European Prospective Investigation into Cancer and Nutrition (EPIC) study. The American journal of clinical nutrition. 2012; 96:1398-1408.

89. Rossi M, Garavello W, Talamini R, Negri E, Bosetti C, Dal Maso L, Lagiou P, Tavani A, Polesel J, Barzan L. Flavonoids and the risk of oral and pharyngeal cancer: a case-control study from Italy. Cancer Epidemiology Biomarkers \& Prevention. 2007; 16:1621-1625.

90. Garavello W, Rossi M, McLaughlin J, Bosetti C, Negri E, Lagiou P, Talamini R, Franceschi S, Parpinel M, Dal Maso L. Flavonoids and laryngeal cancer risk in Italy. Annals of oncology. 2007.

91. Lagiou P, Rossi M, Lagiou A, Tzonou A, La Vecchia C, Trichopoulos D. Flavonoid intake and liver cancer: a casecontrol study in Greece. Cancer Causes \& Control. 2008; 19:813-818.

92. Zamora-Ros R, Fedirko V, Trichopoulou A, González CA, Bamia C, Trepo E, Nöthlings U, Duarte-Salles T, Serafini M, Bredsdorff L. Dietary flavonoid, lignan and antioxidant capacity and risk of hepatocellular carcinoma in the European prospective investigation into cancer and nutrition study. International journal of cancer. 2013; 133:2429-2443.

93. Christensen KY, Naidu A, Parent M-É, Pintos J, Abrahamowicz M, Siemiatycki J, Koushik A. The risk of lung cancer related to dietary intake of flavonoids. Nutrition and cancer. 2012; 64:964-974.

94. Cutler GJ, Nettleton JA, Ross JA, Harnack LJ, Jacobs DR, Scrafford CG, Barraj LM, Mink PJ, Robien K. Dietary flavonoid intake and risk of cancer in postmenopausal women: the Iowa Women's Health Study. International journal of cancer. 2008; 123:664-671.

95. Lagiou P, Samoli E, Lagiou A, Katsouyanni K, Peterson J, Dwyer J, Trichopoulos D. Flavonoid intake in relation to lung cancer risk: case-control study among women in Greece. Nutrition and cancer. 2004; 49:139-143. 
96. Rossi M, Negri E, Lagiou P, Talamini R, Dal Maso L, Montella M, Franceschi S, La Vecchia C. Flavonoids and ovarian cancer risk: a case-control study in Italy. International journal of cancer. 2008; 123:895-898.

97. Arem H, Bobe G, Sampson J, Subar A, Park Y, Risch H, Hollenbeck A, Mayne S, Stolzenberg-Solomon R. Flavonoid intake and risk of pancreatic cancer in the National Institutes of Health-AARP Diet and Health Study Cohort. British journal of cancer. 2013; 108:1168-1172.

98. Rossi M, Lugo A, Lagiou P, Zucchetto A, Polesel J, Serraino D, Negri E, Trichopoulos D, La Vecchia C. Proanthocyanidins and other flavonoids in relation to pancreatic cancer: a case-control study in Italy. Annals of oncology. 2012; 23:1488-1493.

99. Bosetti C, Bravi F, Talamini R, Parpinel M, Gnagnarella P, Negri E, Montella M, Lagiou P, Franceschi S, La Vecchia C. Flavonoids and prostate cancer risk: a study in Italy. Nutrition and cancer. 2006; 56:123-127.
100. Wang Y, Stevens VL, Shah R, Peterson JJ, Dwyer JT, Gapstur SM, McCullough ML. Dietary flavonoid and proanthocyanidin intakes and prostate cancer risk in a prospective cohort of US men. American journal of epidemiology. 2014; 179:974-986.

101. Bosetti C, Rossi M, McLaughlin JK, Negri E, Talamini R, Lagiou P, Montella M, Ramazzotti V, Franceschi $\mathrm{S}$, LaVecchia C. Flavonoids and the risk of renal cell carcinoma. Cancer Epidemiology Biomarkers \& Prevention. 2007; 16:98-101.

102. Xiao Q, Park Y, Hollenbeck AR, Kitahara CM. Dietary flavonoid intake and thyroid cancer risk in the NIH-AARP diet and health study. Cancer Epidemiology Biomarkers \& Prevention. 2014; 23:1102-1108.

103. Rossi M, Edefonti V, Parpinel M, Lagiou P, Franchi M, Ferraroni M, Decarli A, Zucchetto A, Serraino D, Dal Maso L. Proanthocyanidins and other flavonoids in relation to endometrial cancer risk: a case-control study in Italy. British journal of cancer. 2013; 109:1914-1920. 